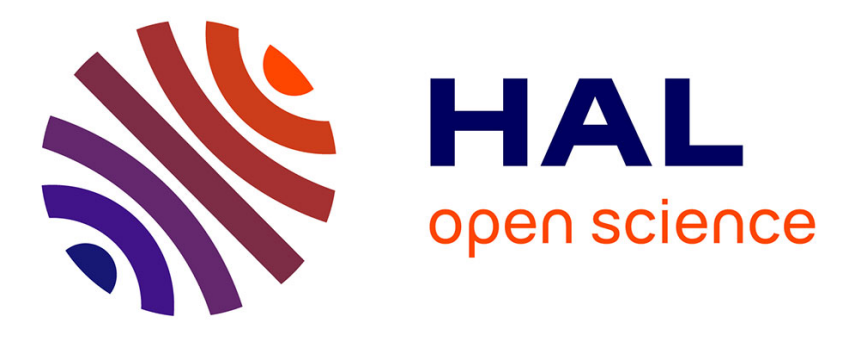

\title{
Scaling-law-based metamodels for the sizing of mechatronic systems
}

Marc Budinger, Jean-Charles Passieux, Christian Gogu, Amine Fraj

\section{To cite this version:}

Marc Budinger, Jean-Charles Passieux, Christian Gogu, Amine Fraj. Scaling-law-based metamodels for the sizing of mechatronic systems. Mechatronics, 2014, 24 (7), pp.775-787. 10.1016/j.mechatronics.2013.11.012 . hal-00949210

\section{HAL Id: hal-00949210 https://hal.science/hal-00949210}

Submitted on 21 Feb 2014

HAL is a multi-disciplinary open access archive for the deposit and dissemination of scientific research documents, whether they are published or not. The documents may come from teaching and research institutions in France or abroad, or from public or private research centers.
L'archive ouverte pluridisciplinaire HAL, est destinée au dépôt et à la diffusion de documents scientifiques de niveau recherche, publiés ou non, émanant des établissements d'enseignement et de recherche français ou étrangers, des laboratoires publics ou privés. 


\title{
Scaling-law-based metamodels for the sizing of mechatronic systems
}

\author{
Marc Budinger ${ }^{1}$, Jean-Charles Passieux, Christian Gogu, Amine Fraj \\ Université de Toulouse, INSA/UPS, Institut Clément Ader, Toulouse, 31077, France.
}

\begin{abstract}
This paper presents a new metamodel form and associated construction procedure adapted to the sizing tasks of mechatronics systems. This method of meta-modeling uses scaling laws to extract compact forms of design models from local numerical simulations (FEM). Compared to traditional metamodels (polynomial response surfaces, kriging radial basis function) the scaling-law-based metamodels have the advantage of a light, compact form and good predictive accuracy over a wide range of the design variables (several orders of magnitude). The general regression process is first explained and then illustrated on different examples: a purely numerical test function, a limited angle electromagnetic actuator and a flexible mechanical hinge.
\end{abstract}

Keywords: metamodels, surrogate modelling, scaling laws, Buckingham theorem, components sizing, preliminary/conceptual design.

\section{NOTATION}

$\begin{array}{ll}\text { CAD } & \text { Computer Aided Design } \\ \text { FEM } & \text { Finite Element Method } \\ \text { 3D } & \text { Three-dimensional } \\ \text { LAT } & \text { Limited Angle Torque } \\ \text { DoE } & \text { Design of Experiments } \\ \text { LHC } & \text { Latin HyperCube } \\ \text { SLAWMM } & \text { Scaling-LAW-based MetaModels } \\ \text { std } & \text { Standard deviation of a variable } \\ \text { mean } & \text { Mean value of a variable }\end{array}$

\section{INTRODUCTION}

A mechatronic system [1] [2] expands the capabilities of conventional mechanical systems through the integration of different technological areas (Figure 1) around:

- a power transmission part, which is a combination of components from mechanical, electrical, power electronics or fluid power technologies;

- an information processing part, which is a combination of electronics, instrumentation, automatic, signal processing, and information technologies.

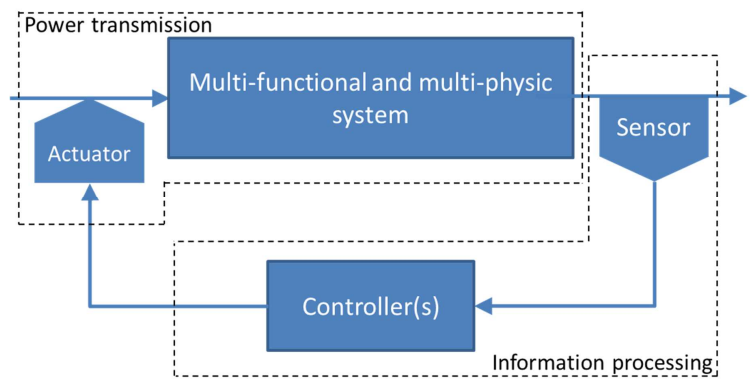

Figure 1 - Mechatronic system (based on reference [3])

1 Corresponding author: Tel.: (+33) 561559960; Fax: (+33) 561559950; E-mail: marc.budinger@insa-toulouse.fr 
The references [3] and [4] highlight that the design of such multi-domain systems require different modeling layers as represented Figure 2:

- A mechatronic layer, to take into account the functional and physical coupling between components. This level of modeling is usually done using 0D-1D models [3] also called lumped parameter models represented by algebraic equations, ordinary differential equations (ODE) or differential algebraic equations (DAE) [5].

- A specific domain layer, to describe the performance limits and parameters necessary in the previous layer, based on a geometric representation. The specific domain phenomena are generally represented through partial differential equations (PDE). This level of modeling can be achieved, for simplified geometries using analytical models or, for complex 2D and 3D geometries, using numerical approximations like finite element method (FEM) for instance.

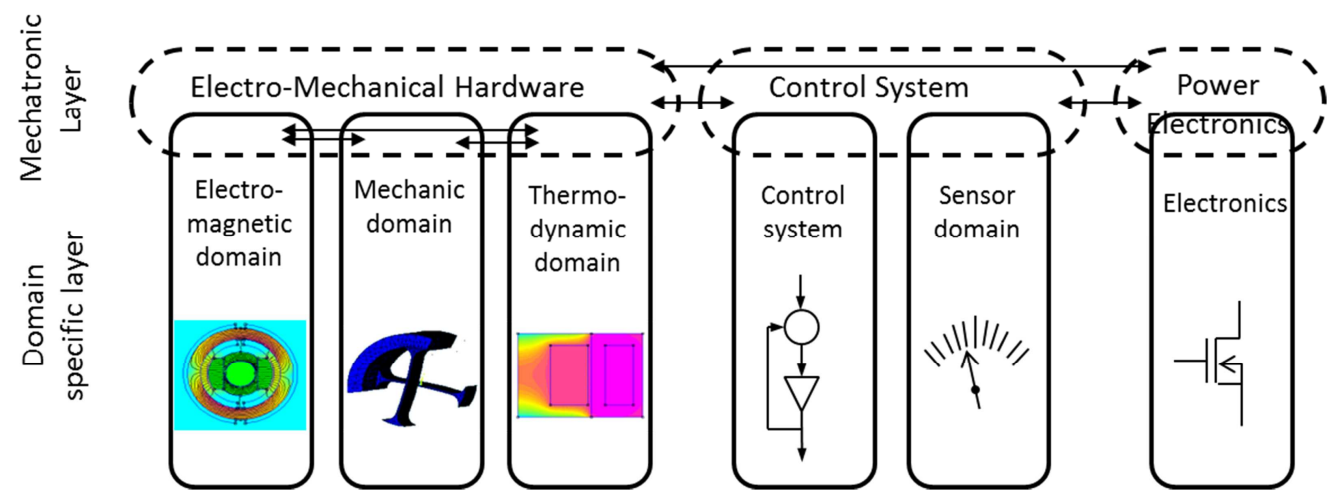

Figure 2 - Hierarchical design models (based on reference [4])

The design of the power part with a system integrator's point of view should allow to optimally size and specify the components of multiple technologies [6] interacting together. This system level design, distinct of component design, needs to represent in the mechatronic layer the key informations of the specific domain layer with dedicated models [7] [8] [9]. The latter enables the designer to take multiple design constraints into account easily. They are referred to as "estimation models" in this paper. They directly and explicitly link a few primary characteristics, such as overall dimensions of components, to the secondary characteristics needed for the sizing [9] and optimization [10]. The capacities required of these estimation models are: to present a form that is simple to handle and to implement in different calculation tools, to lend themselves to easy analytical manipulations, and to be reusable in an area slightly different from the one where they were initially employed. To satisfy these constraints, simplified analytical models are often used [11] [12]. Among them, scaling laws have proved effective to represent a physical phenomenon over wide ranges of variation [13] [8]. However, these models are valid under certain conditions, among which one can mention geometry and material similarities, and uniqueness of the driving physical phenomenon. For the system designer the models should be as predictive as possible. Detailed finite element models, able to precisely predict the physical phenomena are still too time-consuming in such a context. Despite a recent thrust of work on model order reduction [14] [15], the computational cost of finite element models remains prohibitive in the preliminary design phase. The use of metamodelling techniques [16] [17] [18] is thus interesting for this purpose. In this paper, we propose an enhanced power law type model based on metamodels to represent the models of the specific domain layer (Figure 2) into the mechatronic layer.

The second section of this paper will show the interest of scaling laws to establish the estimation models needed by the system designer. In the third section, a metamodeling method based on scaling laws will be proposed to extract simple, global expressions of estimation models from local numerical simulations (FEM). The regression process is first illustrated with a mathematical function and then with two examples of mechatronic components from different domains: a limited-angle electromagnetic actuator and a flexible mechanical hinge. Noise effects and comparisons with classical metamodelling techniques are illustrated through these examples. 


\section{ESTIMATION MODELS WITH SCALING LAWS}

\subsection{Scaling laws and Buckingham theorem}

Scaling laws based on dimensional analysis, also called similarity laws or allometric models, have been very successfully used throughout the past decades for solving scientific and engineering problems and for presenting results in a compact form. In the design of mechatronic systems scaling laws [8] [13], allow estimation models to be obtained from a single reference component by using three main modeling assumptions:

a. Material similarity: all material and physical properties are assumed to be identical to those of the component as the reference;

b. Geometric similarity: the ratio of all the lengths of the component under consideration to all the lengths of the reference component is constant;

c. Uniqueness of design driver: only one main dominant physical phenomenon drives the evolution of the secondary characteristic $y$.

The mathematical form of scaling laws is a power law:

$$
y=k L^{a}
$$

With $y$ the secondary characteristic to be estimated, $L$ the main dimension of the component, and $k$ and $a$ constants. For simplicity of notation, in this article, $k$ means a constant coefficient which may have different numerical values in the different equations.

This form and the conditions for obtaining equation (1) are demonstrated here using the Buckingham theorem [19] [20] [21]. An estimation model seeks to identify a relationship between $2+n+m$ parameters:

$$
f(y, \underbrace{L, d_{1}, d_{2}, \ldots, d_{n}}_{1+n}, \underbrace{p_{1}, \ldots, p_{m}}_{m})=0
$$

with:

- 1 parameter corresponding to the secondary characteristic $y$ to be estimated;

- $\quad l+n$ parameters characterizing the geometrical dimensions $L$ and $d_{i}$;

- $m$ parameters characterizing physical and material properties $p_{i}$;

Depending on the number of physical units $u$ (e.g. $\mathrm{m}, \mathrm{kg}$, s, etc.) involved in the problem, this relationship can be rewritten using dimensionless parameters $\pi_{i}$ :

$$
f^{\prime}(\pi_{y}, \underbrace{\pi_{1}, \pi_{2}, \ldots, \pi_{n}}_{n}, \underbrace{\pi_{p 1}, \ldots, \pi_{p m \prime}}_{m^{\prime}})=0
$$

where:

$$
\begin{gathered}
\pi_{y}=y L^{a_{L}} \prod_{d_{i}} p_{i}^{a_{i}} \\
\pi_{p i}=L^{a_{L p i}} \prod p_{j}^{a_{j}}
\end{gathered}
$$

The number $m$ ', usually smaller than $m$, depends on the number of physical units $u$ as expressed by the Buckingham theorem:

$$
m^{\prime}=1+m-u
$$

If only one main simple physical phenomenon drives the evolution of the secondary characteristic $y$, the number $m$ ' is often equal to zero. If $m^{\prime}$ is not equal to zero, the remaining dimensionless numbers can generally be expressed through ratios of material properties with similar units. These $m$ ' dimensionless number thus do not depend on $L$. With this condition and the first two assumptions a. and b., the $\pi_{i}$ and $\pi_{p i}$ dimensionless numbers are constant and it follows that:

$$
\pi_{y}=y L^{a_{L}} \prod p_{i}^{a_{i}}=\text { constant }
$$

which gives relation (1) if we assume the material or physical properties $p_{i}$ to be constant during the sizing. 


\subsection{Examples of scaling laws}

To illustrate the construction and use of such laws, two examples will be given here. These examples address conventional components of mechatronic systems: brushless motors and bearings. We assume here that the main design criterion for the motor is the winding temperature. The dominant thermal phenomenon will be assumed to be convective. The bearings are for their part designed to withstand a maximum mechanical stress.

For the brushless motors, equations (2), (3) and (1) become the following:

- For thermal aspects: the current density $J$ can be linked to the dimensions through

$$
f(J, \underbrace{L, d_{1}, d_{2}, \ldots, d_{n}}_{1+n}, \underbrace{\rho, \theta, h}_{m})=0
$$

which, according to the Buckingham theorem, leads to

and

$$
f^{\prime}(\frac{\rho J^{2} L}{h \theta}, \underbrace{\frac{d_{1}}{L}, \frac{d_{2}}{L}, \ldots, \frac{d_{n}}{L}}_{n})=0 \text { where } m^{\prime}=0
$$

$$
J=k L^{-0,5}
$$

- For magnetic aspects : the torque $T$ can be linked to dimensions and current density through

$$
f(T, \underbrace{L, d_{1}, d_{2}, \ldots, d_{n}}_{1+n}, \underbrace{J, B_{r}}_{m})=0
$$

which, according to the Buckingham theorem, leads to

and

$$
f^{\prime}(\frac{T}{J B_{r} L^{4}}, \underbrace{\frac{d_{1}}{L}, \frac{d_{2}}{L}, \ldots, \frac{d_{n}}{L}}_{n})=0 \text { where } m^{\prime}=0
$$

$$
T=k J L^{4}
$$

with : $J$ the current density, $L$ the length of the motor, $d_{i}$ other geometrical dimensions, $\rho$ the resistivity of the copper, $\theta$ the maximal temperature rise for the winding insulation, $h$ the convection coefficient, $B_{r}$ the remanent induction of the permanent magnet, and $T$ the electromagnetic torque.

Combining these two aspects provides an estimate of torque depending on motor size:

$$
T=k L^{3,5}
$$

The assumptions a. and $\mathrm{b}$. allow the motor weight to be estimated:

$$
M=k L^{3}=k T^{3 / 3,5}
$$

With the same approach, the weight of the bearing can be estimated from their load-bearing capacity $C$ :

$$
C=k L^{2} \text { and } M=k L^{3}=k C^{3 / 2}
$$

More details and examples of the development of such laws for mechanical and electromechanical components are given in [8]. Figure 3 and Figure 4 compare these relationships to data from industrial catalogues, and show that scaling laws can provide good fits for the quantities of interest of such components 


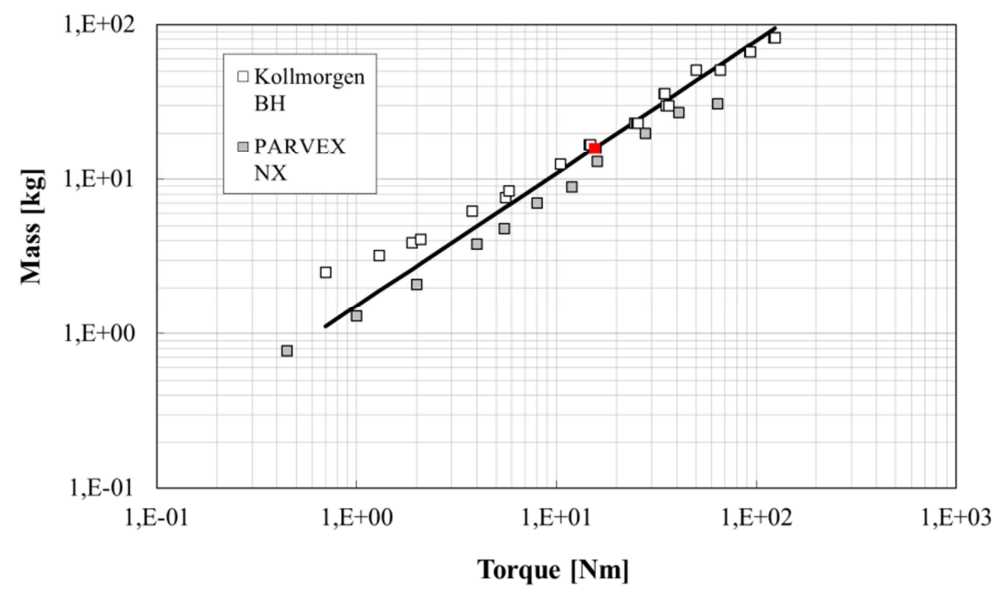

Figure 3 - Brushless motor masses according to the nominal torque.

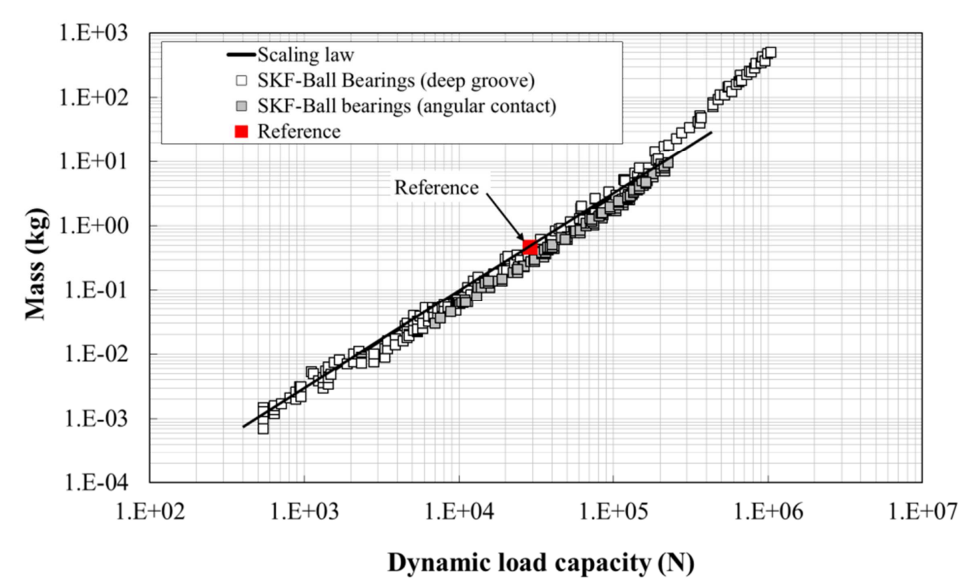

Figure 4 - Bearing masses according to the load capacity.

\subsection{Interests and limitations of scaling laws}

Scaling laws have assets that make them attractive for the design of mechatronic systems [22] [23] [9]. Their simple form makes them easy to manipulate and customize as they require only one reference to determine the multiplier coefficient $k$, the power coefficient $a$ being determined by the physical phenomena. They have a monotonous progression valid over a wide range of sizes (several orders of magnitude) which avoids the risk of possible mathematical aberrations of metamodels used outside their construction bounds.

However they have some limitations. Although the similarity of the materials can be easily verified for a given technology, the geometric similarity is not necessarily verified or sought. For motors (Figure 3), this point mainly explains the estimation errors of scaling laws in Figure 3. Manufacturers have a tendency to use the same motor diameter (i.e. the same iron sheet) for different lengths and torques. Obtaining a scaling law also requires a dominant physical phenomenon. For example, in the case of the electric motor, if the conductive heat transfer phenomenon cannot be neglected, relations (9) and (10) should take the form:

$$
f(J, \underbrace{L, d_{1}, d_{2}, \ldots, d_{n}}_{1+n}, \underbrace{\rho, \theta, h, \lambda_{1}, \lambda_{2}, \ldots}_{m})=0
$$

which gives

$$
f^{\prime}(\frac{\rho J^{2} L}{h \theta}, \underbrace{\frac{d_{1}}{L}, \frac{d_{2}}{L}, \ldots, \frac{d_{n}}{L}}_{n}, \underbrace{\frac{\lambda_{1}}{h L}, \frac{\lambda_{2}}{h L}, \ldots}_{m \prime})=0
$$


with $\lambda_{i}$ the thermal conductivities of the various materials of the motor.

In this case, either a global scaling law would have been impossible to establish or its domain of validity would have been smaller.

\section{SCALING LAWS BASED ON REGRESSION}

The objective of this section is to show how it is possible to increase the use and validity of scaling laws through appropriate regression techniques as proposed by our method.

\subsection{Form of models}

To maintain the physical meaning and benefits of scaling laws, the regression model will be based on the power form (1). However, to eliminate assumptions b. (geometric similarity) and c. (uniqueness of design driver), estimation models of the following, more general form will be sought:

$$
y=f\left(L, \pi_{1}, \pi_{2}, \ldots\right)=k\left(\pi_{1}, \pi_{2}, \ldots\right) L^{a\left(\pi_{1}, \pi_{2}, \ldots\right)} \text { with } \pi_{i}=\frac{d_{i}}{L}
$$

where :

- $\quad y$ is the parameter to be estimated;

- $\quad L$ is the main dimension of the system;

- $d_{i}$ are the secondary dimensions of the system;

- $\pi_{i}$ are the dimensionless numbers representative of form factors of the system.

With geometrical similarity, the form factors are constant $\left(\pi_{i}=C^{s t}\right)$ and the form (20) simplifies into the classic scaling law form (1). The desired form of model (20) lends itself to direct regression less easily than polynomial response surfaces [24], radial basis functions [16] [25], or kriging [26] [27] directly on the parameter $y$. The objective of the coming sections is to give an approach to determine the shapes of functions $k\left(\pi_{1}, \pi_{2}, \ldots\right)$ and $a\left(\pi_{1}, \pi_{2}, \ldots\right)$ representative of multiplier and power coefficients.

\subsection{Regression process}

Figure 3 describes the overall meta-modelling process proposed to carry out regressions of form (20). The three main steps of the process are described below and the details of each step are described in the following subsections.

- $\quad$ Step 1 -data generation: the objective is first to generate the combinations of variables $L$ and $\pi_{i}$ to be simulated. With deterministic computer experiments, these sample points should be chosen to fill the design space. Once the DoE is defined, a call is then made to the finite element codes, or any other sizing code, to obtain the desired data $y$. The data $y$ are then projected according to form (20) by calculating the coefficients $k$ and $a$ for each configuration $\pi_{i}$ of form factor parameters.

- Step 2 - study of the general shape of the function: the evolution of each coefficient $k$ and $a$ is then analysed to determine the overall shape to be imposed on function (20) through the choice of the forms of functions $k\left(\pi_{1}, \pi_{2}, \ldots\right)$ and $a\left(\pi_{1}, \pi_{2}, \ldots\right)$. These forms of functions cannot be initially assumed and depend on each problem.

- $\quad$ Step 3 - building of the metamodel: based on the function forms selected in the previous step, a regression process is applied to simulation data. A comparison of the results predicted using Eq. (20) with the initial data $y$ can then validate the quality of these regressions.

Even though the model form of Eq. (20) cannot be expressed by linear combination of basis functions, some of its characteristics are interesting and will be used for the proposed meta-modeling process. The following subsections describe the choices made here for each of these steps according to these characteristics. 


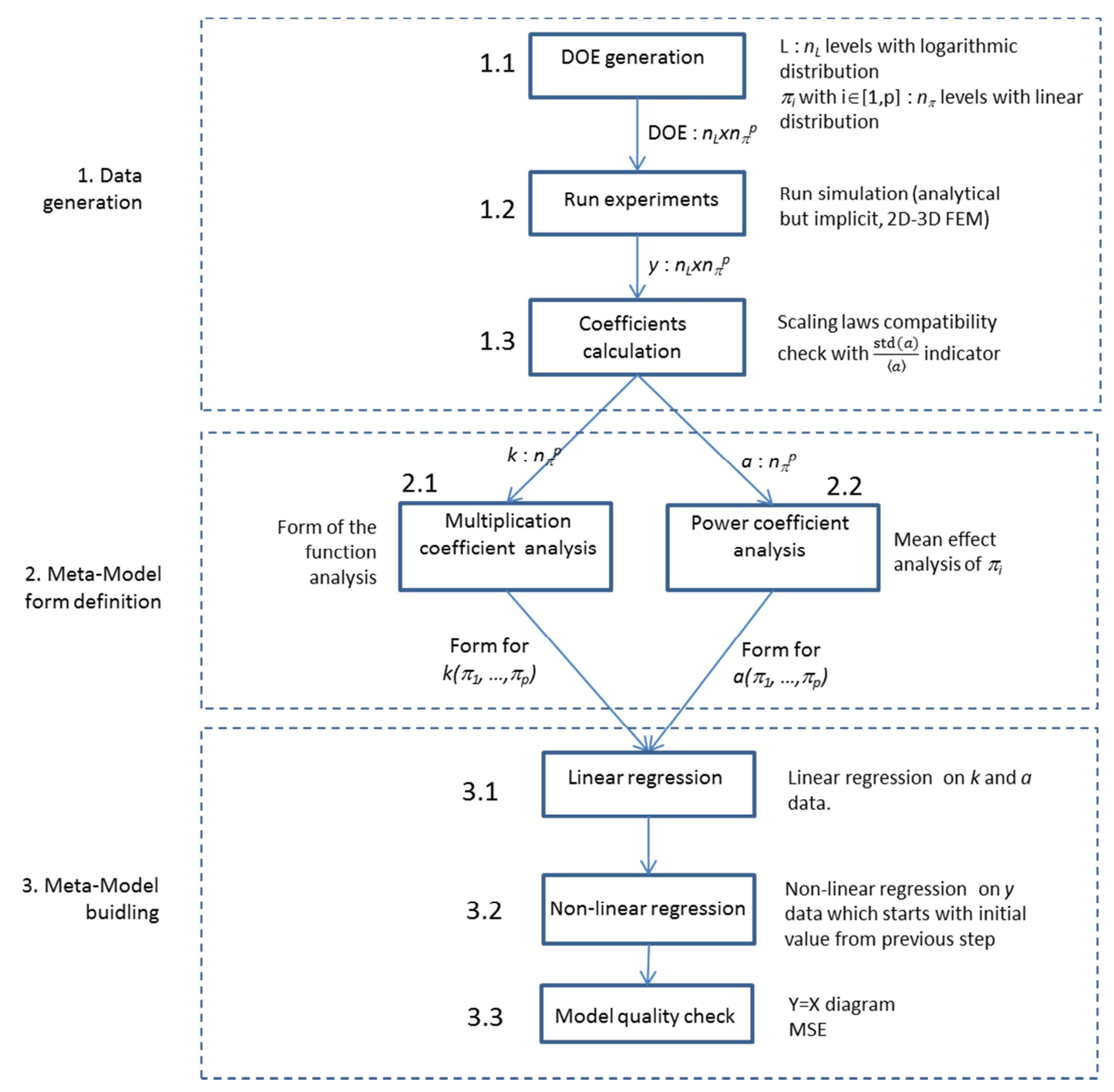

Figure 5 - Meta-modeling process

\subsubsection{Step 1 - Data generation}

The range of variation for the parameter $L$ is potentially very large and is potentially much smaller for the shape parameters $\pi_{i}$. The proposed process will use a DoE (design of experiments) which is logarithmically distributed on $n_{L}$ levels for the parameter $L$ and linearly distributed on $n_{\pi}$ levels for the $p$ parameters $\pi_{i}$. This simple DoE enables the design space to be filled. This DoE (step 1.1), mainly similar to a full factorial design, requires at least three levels for each parameter in order to calculate (steps 1.3) and analyse (steps 2.1 and 2.2) coefficients $k$ and $a$.

According to this DOE, calls are then made (step 1.2) to the finite element codes, or any other sizing code, to obtain the desired data $y$. The final number of data $y$ is $n_{L} . n_{\pi}^{p}$. The conventional regression techniques use these simulation data directly. Here in order to adapt the data to form (20) and as a power law can be represented linearly in logarithmic-scale graphs, the projection of the data $y$ onto the coefficients $a$ and $k$ (step 1.3 of Figure 5 ) is done in a logarithmic plane plotting the $y$ according to $L$ data for each combinations of the form factors $\pi_{i}$. Figure 6 represents the graphs where, for each combination of coefficients $\pi_{i}$, the evolution of $y$ according to $L$ follows a power law and is therefore described by a straight line in a logarithmic plane. The algorithm computes the slopes $a$ of the segments between two consecutive values of $L$ and returns the mean average slope mean $(a)$ for each combinations of $\pi_{i}$. The multiplier coefficient $k$ is calculated for each curve by minimizing the least square error for the average slope. In order to check that the data are well fitted by power laws, a quality criterion is also calculated. This criterion expressed by the relation $\operatorname{std}(a) /$ mean $(a)$ is usually very close to zero and shows 
that the problem is well expressed by using a scaling law for a given configuration of form factors. After this step, the $n_{L} \cdot n_{\pi}^{p}$ values $y$ are transformed into $n_{\pi}^{p}$ values vectors for all coefficients $a$ and $k$.

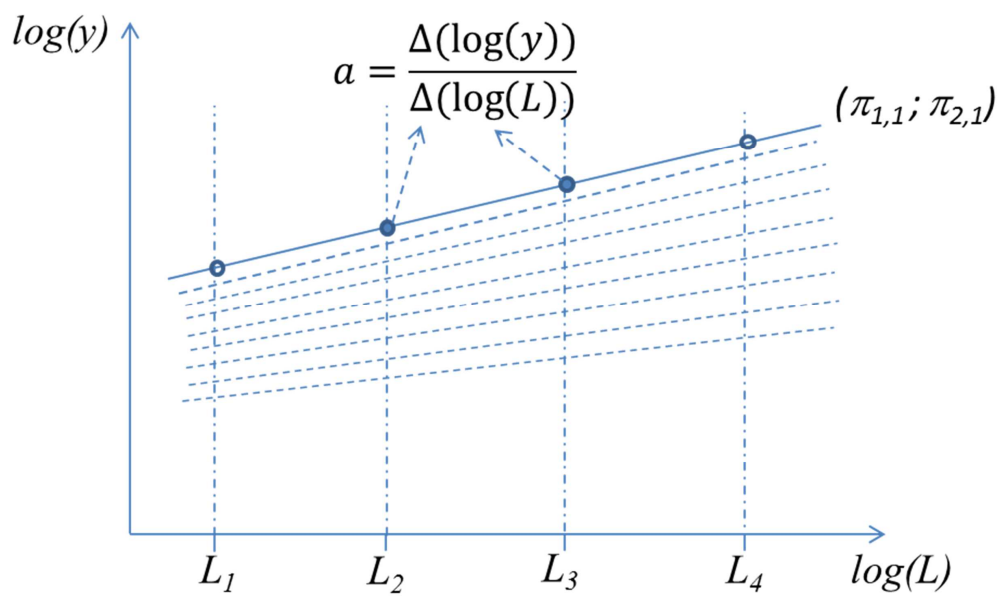

Figure 6 - Power law coefficients calculation

\subsubsection{Step 2 - Metamodel form definition}

The objective of this step is to determine the form that can be given to functions $a\left(\pi_{1}, \pi_{2}, \ldots\right)$ and $k\left(\pi_{1}, \pi_{2}, \ldots\right)$.

The power coefficient $a\left(\pi_{1}, \pi_{2}, \ldots\right)$ of scaling laws is constant if the physical phenomenon that drives the design of the system does not depend on the form factors $\pi_{i}$. If the physical phenomenon switches from one to another (e.g. convection to conduction for thermal transfer), the power coefficient is a function that changes between 2 extreme values. If the switch between the two physical phenomena is progressive (often the case for components of mechatronics systems) then the surface $a\left(\pi_{1}, \pi_{2}, \ldots\right)$ will be characterized by a small number of peaks and valleys and can potentially be well approximated by a polynomial function. It remains to determine the exact form of the polynomial function. Since all the parameters $\pi_{i}$ do not usually affect the power coefficient it is useful to conduct a sensitivity analysis at this point. Step 2.2 thus corresponds to a sensitivity analysis, which is conducted here by performing a linear regression after normalization between -1 and +1 of parameters $\pi_{i}$. The analysis of the regression coefficients leads to the quantification of the average influence of each parameter. Only relevant parameters will be considered in the final expression of $a\left(\pi_{1}, \pi_{2}, \ldots\right)$. Note that any other technique of sensitivity analysis could be used here (e.g. ANOVA [28] or Sobol's indices [29]).

The multiplication coefficient $k\left(\pi_{1}, \pi_{2}, \ldots\right)$ of scaling laws can often take the form of products of functions, some of which may also be expressed in the form of power laws. Step 2.1 (Figure 5) analyses these options by using a logarithmic plane again as the functions that can be decomposed into products are represented by parallel curves in logarithmic-scale graphs (Figure 7a). The power laws are represented by straight lines (Figure 7b). If:

$k\left(\pi_{1}, \pi_{2}, \ldots\right)=f\left(\pi_{1}\right) g\left(\pi_{2}, \ldots\right)$ or $\log \left(k\left(\pi_{1}, \pi_{2}, \ldots\right)\right)=\log \left(f\left(\pi_{1}\right)\right)+\log \left(g\left(\pi_{2}, \ldots\right)\right)$

the evolution of values of $k\left(\pi_{1}, \pi_{2}, \ldots\right)$, a function of $\pi_{1}$ for different $\pi_{i \neq 1}$ configurations, can give 3 possibilities: factorizable (Figure 7a), factorizable with a power law (Figure 7b), or non-factorizable (Figure 7c). This type of analysis can be carried out for each variable $\pi_{i}$ and quantified by indicators describing:

- the possibility of factorizing $k\left(\pi_{1}, \pi_{2}, \ldots\right)$ by a function of $\pi_{i}$. To assess this possibility we introduce the indicator defined by $(\max (E)-\min (E)) /(\max (\log (k)-\min (\log (k))$, where $E$ quantifies the difference between two curves for different values of $\pi_{i}$. If the difference between two curves is constant, this indicator is equal to zero and points out the possibility of factorization.

- the opportunity of expressing this function by a power law. To assess this, we introduce the indicator $(\max (s)-\min (s)) /(\operatorname{mean}(s) \mid$, where $s$ quantifies the slope of a curve. If the slope is constant, the indicator is equal to zero and indicates a power law.

These criteria can be condensed graphically on a plane, as illustrated in Figure 7d, to quickly assess the form that the function $k\left(\pi_{1}, \pi_{2}, \ldots\right)$ should have. 


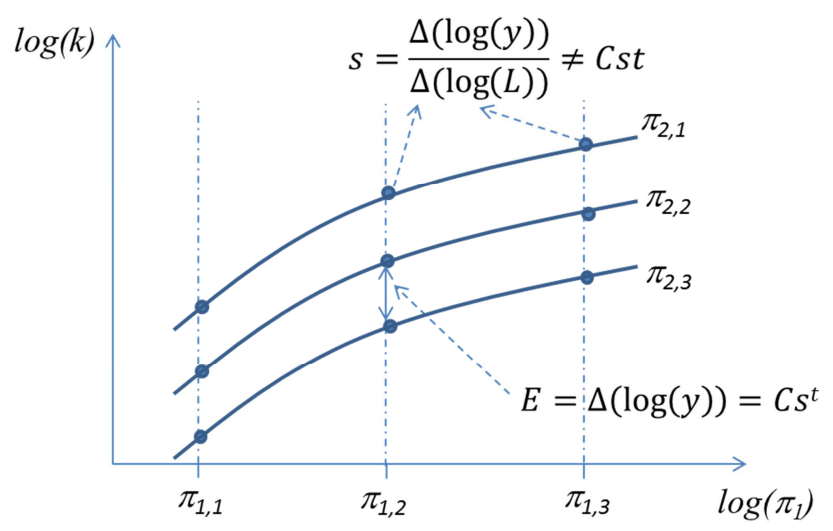

a) Factorizable

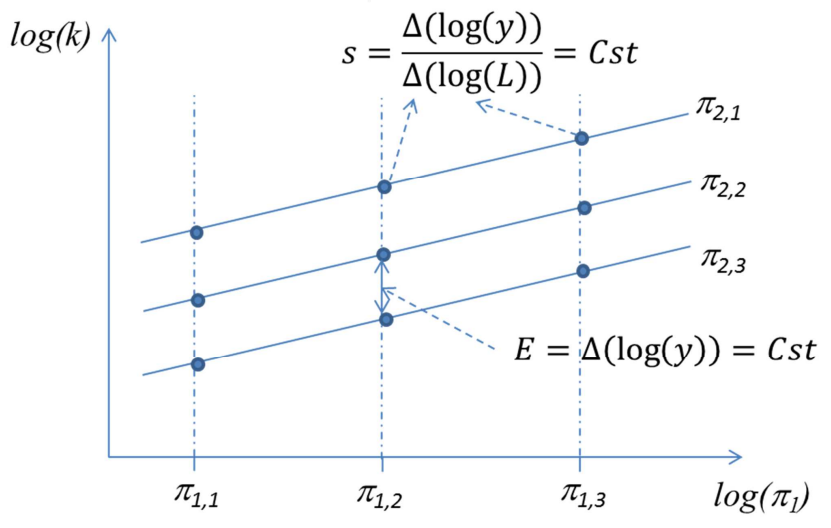

b) Factorizable with a power law

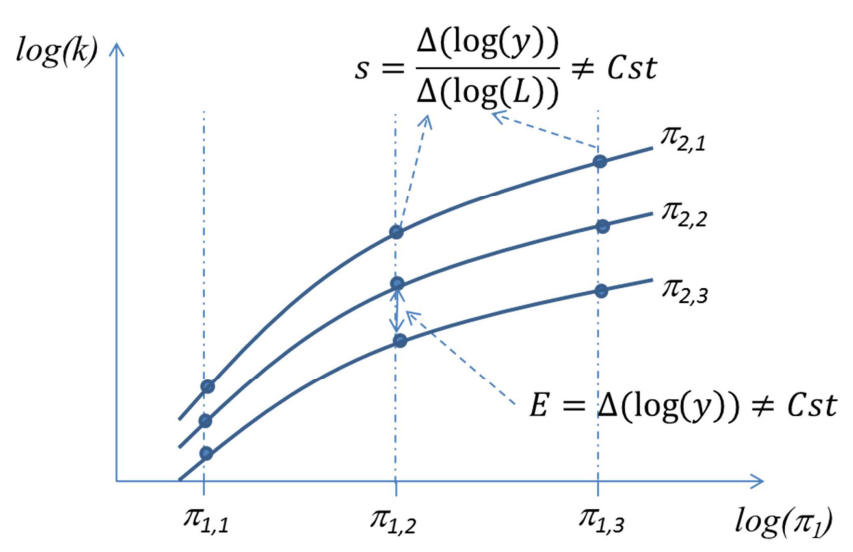

c) Non-factorizable 




d) Indicator map

Figure 7 - Multiplication coefficient form analysis

\subsubsection{Step 3 - Metamodel building}

The last step aims to build the metamodels from data generated in step 1 and according to the forms of functions defined in step 2 . This process of regression is done in two sub-steps:

- The first uses the data $a$ and $k$ (outputs of step 1.3, Figure 5): a least squares polynomial regression, using the most influential parameters, is directly performed on the data $a$ to obtain an approximation for $a\left(\pi_{1}, \pi_{2}, \ldots\right)$. Depending on the decomposition of $k\left(\pi_{1}, \pi_{2}, \ldots\right)$ derived from the indicators of step 2.1 (Figure $7 \mathrm{~d}$ ), the regression is performed on each of the factorizable functions. Power laws are processed first by linear regression in logarithmic planes. Functions that cannot be expressed by power laws are approximated by polynomial functions using least squares regression.

- The second uses y data directly (outputs of step 1.2, Figure 5): this nonlinear regression is initialized with results of the first regression and minimizes relative errors thanks to the lsqnonlin function of Matlab.

Finally a comparison of the two regression functions with respect to the initial data $y$ can validate the quality of these regressions.

\subsection{Example with a test function}

A purely numerical example will help to illustrate and detail the various steps of the process described in Section 4.2. This test function, which uses the form of equation (20), is:

$$
y=\pi_{1}^{-1}\left(4+5 \pi_{2} \pi_{3}\right) \pi_{4}^{3} L^{2+0.1 \pi_{2}}
$$

A numerical uniformly distributed noise can be added to $y$ data in order to test the robustness of the process to the possible computer noise of design codes or to variations due to neglected phenomena. For the first numerical application, this noise is set to $+/-1 \%$ of y data. The different stages of regression, shown from Figure 8 to Figure 11, are:

- Step 1.1, the generation of a design of experiments: $L$ with 4 levels between 1 and $10^{4}$ and each parameter $\pi_{i}$ with 3 levels between respectively .5 and 2, 1 and 10,2 and 7, 1 and 10. The number of simulations and data $y$ is $4.3^{4}=324$.

- Step 1.3, the $y$ data projection: The straight lines (cf. Figure 8) imply the power law nature of the relationship. For the example of Figure 8, the slope $a$ is equal to 3. The deviation from a power law is expressed by a quality indicator, $\operatorname{std}(a) /$ mean $(a)$ as explained in 3.2.1, which has the value of $0.24 \%$ and also appears in Figure 8. Without the parameter $L$ the remaining design of experiments now has $3^{4}=81$ values for each parameter $a$ and $k$. 
- Step 2, a study of the general shape of the function: Figure 9 shows the analysis (step 2.1) of the possible form for the function $k\left(\pi_{1}, \pi_{2}, \ldots\right)$. From indicators presented in Figure 7 and section 4.2, it follows that the parameters $\pi_{1}$ and $\pi_{4}$ are suitable for factorization using power laws. Figure 10 illustrates the sensitivity analysis (step 2.2) to determine parameters influencing the evolution of the $a$ coefficient. Only the parameter $\pi_{2}$ has to be kept here. We thus select an equation with the following form:

$$
y=k\left(\pi_{1}, \pi_{2}, \ldots\right) L^{a\left(\pi_{1}, \pi_{2}, \ldots\right)}=k^{\prime}\left(\pi_{2}, \pi_{3}\right) \pi_{1}^{a_{1}} \pi_{4}^{a_{4}} L^{a\left(\pi_{2}\right)}
$$

with $k^{\prime}\left(\pi_{2}, \pi_{3}\right)$ and $a\left(\pi_{2}\right)$ second order polynomial functions.

- Step 3, the construction of the metamodel based on functions forms selected in the previous step: functions $k^{\prime}\left(\pi_{2}, \pi_{3}\right)$ and $a\left(\pi_{2}\right)$ will be approximated by polynomial function. The first regression allows functions $k\left(\pi_{1}, \pi_{2}, \ldots\right)$ and $a\left(\pi_{1}, \pi_{2}, \ldots\right)$ to be obtained independently by a log plane study (for the power law) or linear regression (for the other function). This first regression (step 3.1) gives :

$y=\left(4.84-0.14 \pi_{2}-0.37 \pi_{3}+5.01 \pi_{2} \pi_{3}+0.01 \pi_{2}^{2}+0.03 \pi_{2}^{2}\right) \pi_{1}^{-0.99} \pi_{4}^{3} L^{\left(2+0.10 \pi_{2}\right)}$

The second non-linear regression (step 3.2) working directly on $y$ and $\pi_{i}$ data enables us to find :

$$
y=\left(3.988+0.002 \pi_{2}+0.002 \pi_{3}+5.001 \pi_{2} \pi_{3}\right) \pi_{1}^{-1} \pi_{4}^{3} L^{\left(2+0.10 \pi_{2}\right)}
$$

For the 2 regressions, the mean square error minimizes the relative and not the absolute errors. The regression quality check shows that less than $1 \%$ of error is achieved with equation (25). Without noise, exact relationship is found directly with the first and second regression.

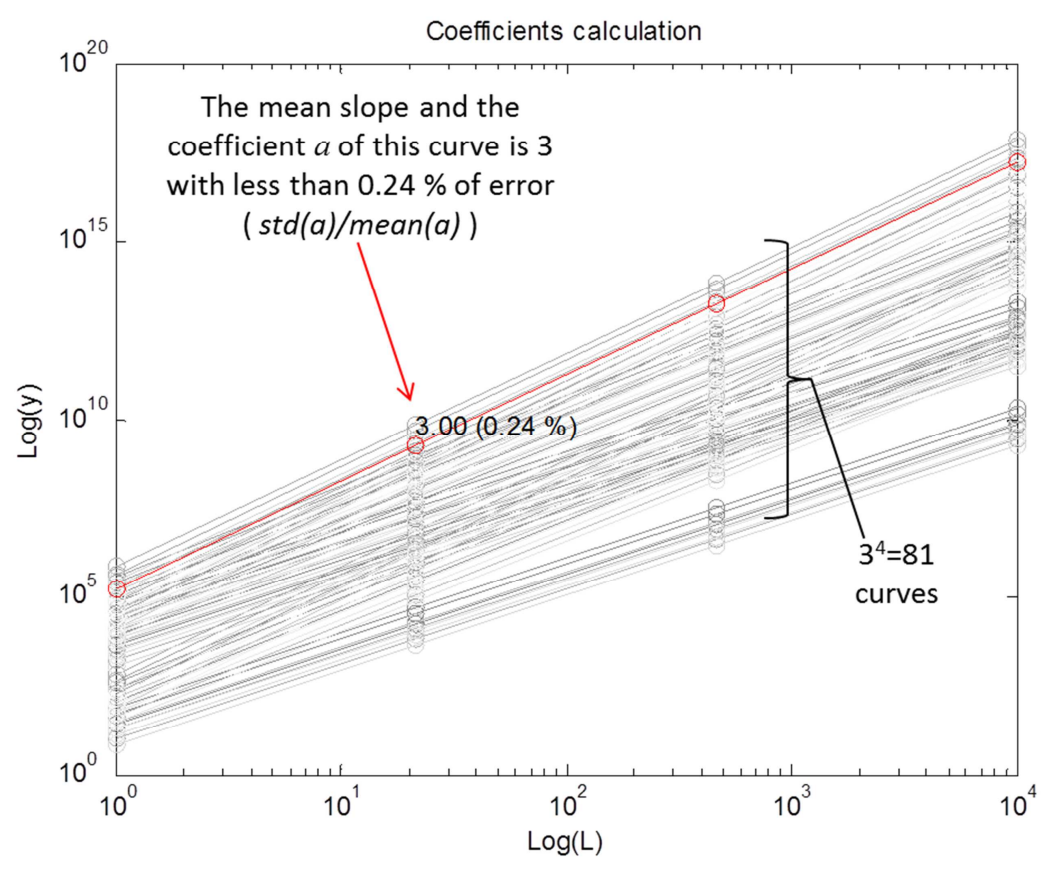

Figure 8-Power law coefficients calculation for the test function 


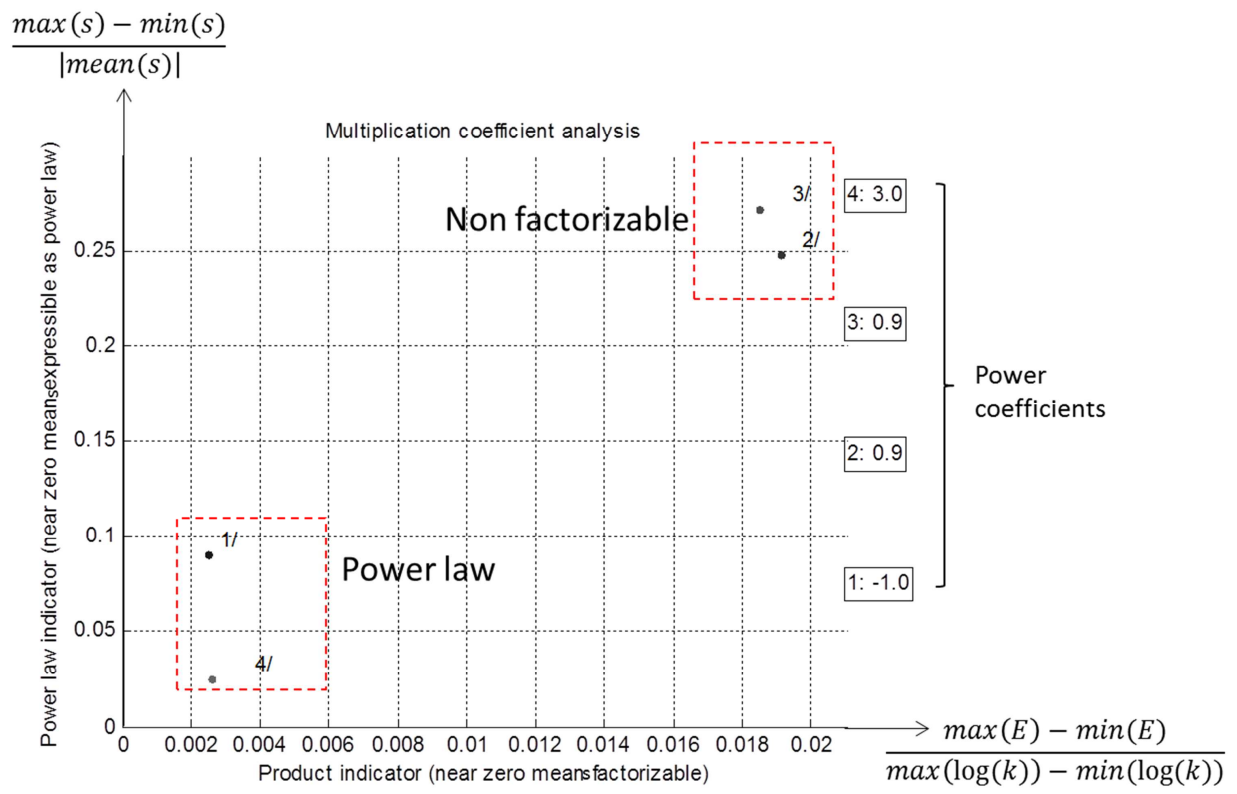

Figure 9 - Form analysis of multiplication coefficient $k$ for the test function

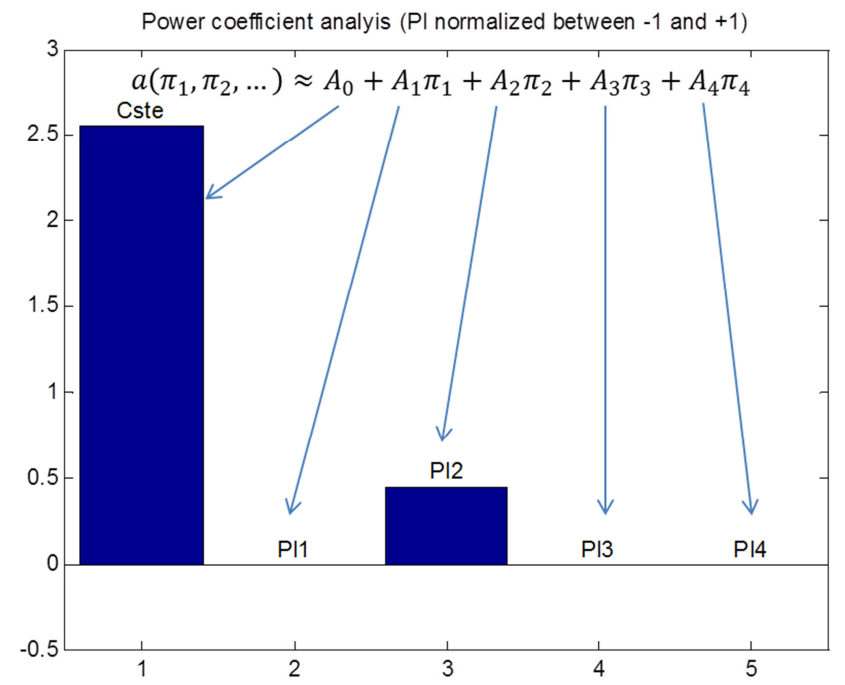

Figure 10 -Sensitivity analysis for the power coefficient a of the test function

Even though numerical simulations are deterministic, errors may exist due to simplifications of the mathematical model or systematic errors of the numerical scheme. To quantify the effect of errors on the proposed process more precisely, we assume here that the error is similar to white noise of amplitude proportional to the quantity of interest $y$. The calculation process was repeated for different values of maximum noise: $0.2,0.5,1,2$ and 4\%. Figure 9 shows the results of step 2.1 and 3.3 for these different levels. Figure 11a) was plotted with 10 runs for each noise level. Figure 11b) quantifies the maximum error found between the expression and the simulation results (with noise) or the pure analytical formula (without noise). From these results we can conclude that:

- $\quad$ Step 3 (regression), with its two regression sub-steps, is quite robust to noise and even decreases the influence of noises. 
- Step 2.1 (determination of the shape of the coefficient $\mathrm{k}$ ) is more sensitive to noise but allows the form of the function to be distinguished for noise levels less than $+/-2 \%$. The FEM simulations should therefore have a meshing quality sufficient to allow for this step.

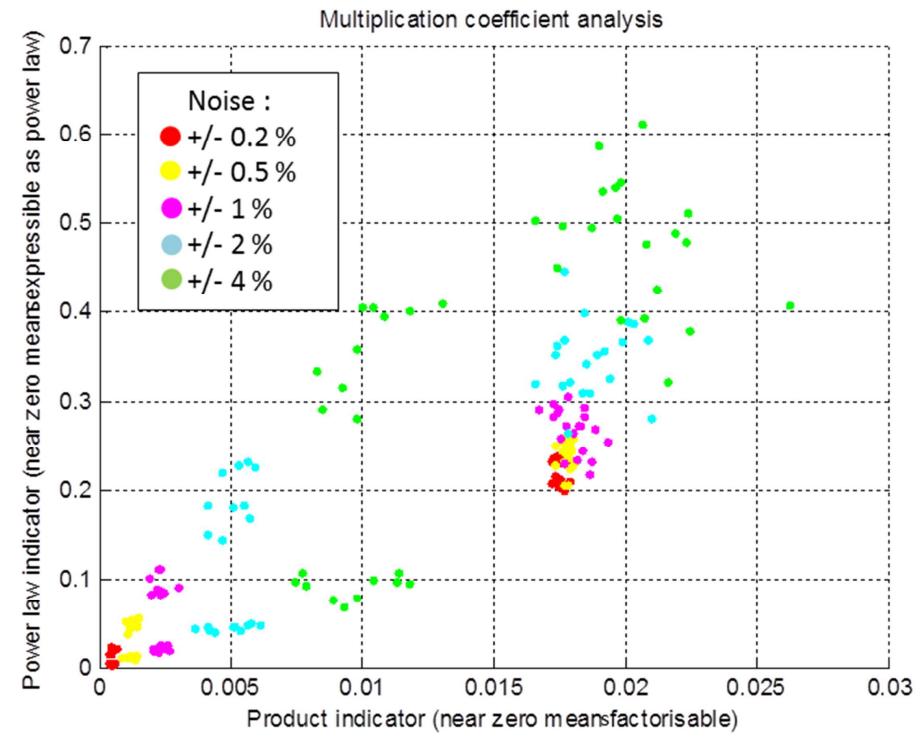

a) Effect of noise on step 2.1

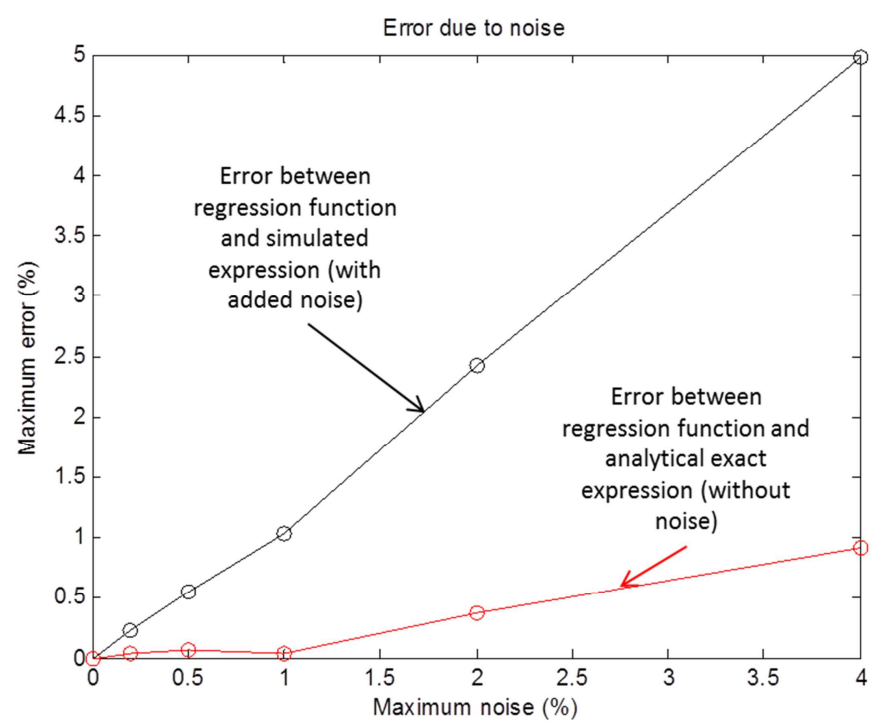

b) Effect of noise on step 3

Figure 11 - Noise effect on indicator map and final regression quality

\subsection{Comparison with other analysis and regression methods}

Reference [16] gives a classification of the different approximation techniques according to 3 criteria: the type of DoE, the choice of model and the model fitting approach. Here, the proposed meta-modelling approach is based on:

- a full factorial DoE with logarithmic distribution of levels distribution for the main dimension $L$ parameter; 
- a model choice based on scaling laws and power laws;

- a fitting approach using mainly linear and non-linear least square regression based on a projection of the data on scaling law coefficients.

Classic regression procedures generally start with a screening step mainly focusing on selection of the input parameters. The process assumes that this selection has been performed thanks to a previous sensitivity analysis or the designer's knowledge. Preliminary analysis, here, is devoted to determining the exact form of the model. Indicators and a graphical synthesis, similar to the Morris method [30], have been proposed for this purpose.

Compared to the classical meta-modeling approaches (polynomial, kriging, radial basis functions, etc) the advantage of the method proposed here lies mainly in its physical justification, demonstrated here by using the Buckingham theorem. A recent review about dimensional analysis and pi-theorem illustrated by an example of application to the modeling of a Flexible Manufacturing System can be found in [21]. Some other authors [31] [32] [33] have also used this theorem with the main objective of reducing the number of parameters to be handled by polynomial regressions. Other authors [34] have used dimensional analysis to determine constraints on power law regressions. Their approach, like other approaches solely based on dimensional analysis, however requires handling of all the parameters representing the dimensions and physical properties. Moreover, the scaling laws employed are pure power laws without the possibility of using other functions to express multipliers or power coefficients.

The approach proposed here does not directly use dimensional analysis but the particular form of the functions representing the physical characteristics of a component subjected to a change in size in the case of particular similarities. Previous studies [35] [20] on similarity have also worked on the development of models valid in the case of non-similarities (called distored models) but have not been applied to numerical simulations within a general framework. The possibility of keeping constant the ratios of some of the dimensions reduces the complexity of representation of the detailed design models. A compact model is then obtained with only the relevant design parameters for the design of mechatronic layer without having to handle all the parameters of the detailed design.

\section{Case Studies}

The objective here is to illustrate the proposed regression process on mechatronic components from different domains. The main focus is placed on the illustration of the regression process and not to on the complete design study of a system. Reference [10] presents a methodology for the optimal preliminary design of electromechanical actuators using a model-based approach with different types of models (estimation, simulation, evaluation and meta-model).

The components considered are:

- an electromagnetic limited angle actuator [36], as shown in Figure 13a from [37], which illustrates the thermal and electromagnetic domains;

- a flexural hinge composed of thin blades, as shown in Figure 13b from [38], which illustrates the structural and mechanical domains.

The regression process could be applied to other types of motor, such as a brushless motor or a voice coil actuator [39], other flexural hinge [39] [38] or any other physical mechatronic components. These 2 components associated with a mirror, a position sensor and control electronic could be used to build up a scanning mechanism [40]. The overall design of such mechatronic systems requires consideration of the various constraints that have a direct impact on the size of the components. These design constraints may come from different types of specifications:

- Performance specifications, particularly in terms of motion range, accuracy or bandwidth: the motion is linked to the rotational stiffness of the hinge and to the torque capability of the LAT actuator; the accuracy and the bandwidth are linked with resonance modes, which depend on the inertia and stiffness of components.

- Endurance specifications, particularly in terms of life time and resistance to extreme stresses: the flexural link should be used at stress levels consistent with these specifications.

These two points are influenced by the environmental mechanical stress (vibration, acceleration) or thermal stress (a function of the type of heat transfer and the ambient temperature). Embedded applications add 
constraints of integration (size, volume or mass). All these constraints can be represented (Figure 12) by design scenarios such as:

a) Thermal and mechanical mission profiles: these mission profiles are representative of the movement of the mirror during scanning. These time simulations can be used to calculate the average electromagnetic torque developed by the LAT. One can note that there exists a coupling of this torque with the rotational stiffness of flexural hinges.

b) Stability analysis: the transfer function of the overall multiphysic system can be used to estimate the closed loop performance especially in terms of bandwidth and stability margins. The indicators will be significantly influenced by the rotational resonance modes.

c) Vibratory environment: transient or frequential profiles for acceleration at the support can be used to represent the aggressive vibratory environment. The transverse or longitudinal modes of resonance are all influenced by the transverse or longitudinal stiffness of the flexible pivots and the mass of components.

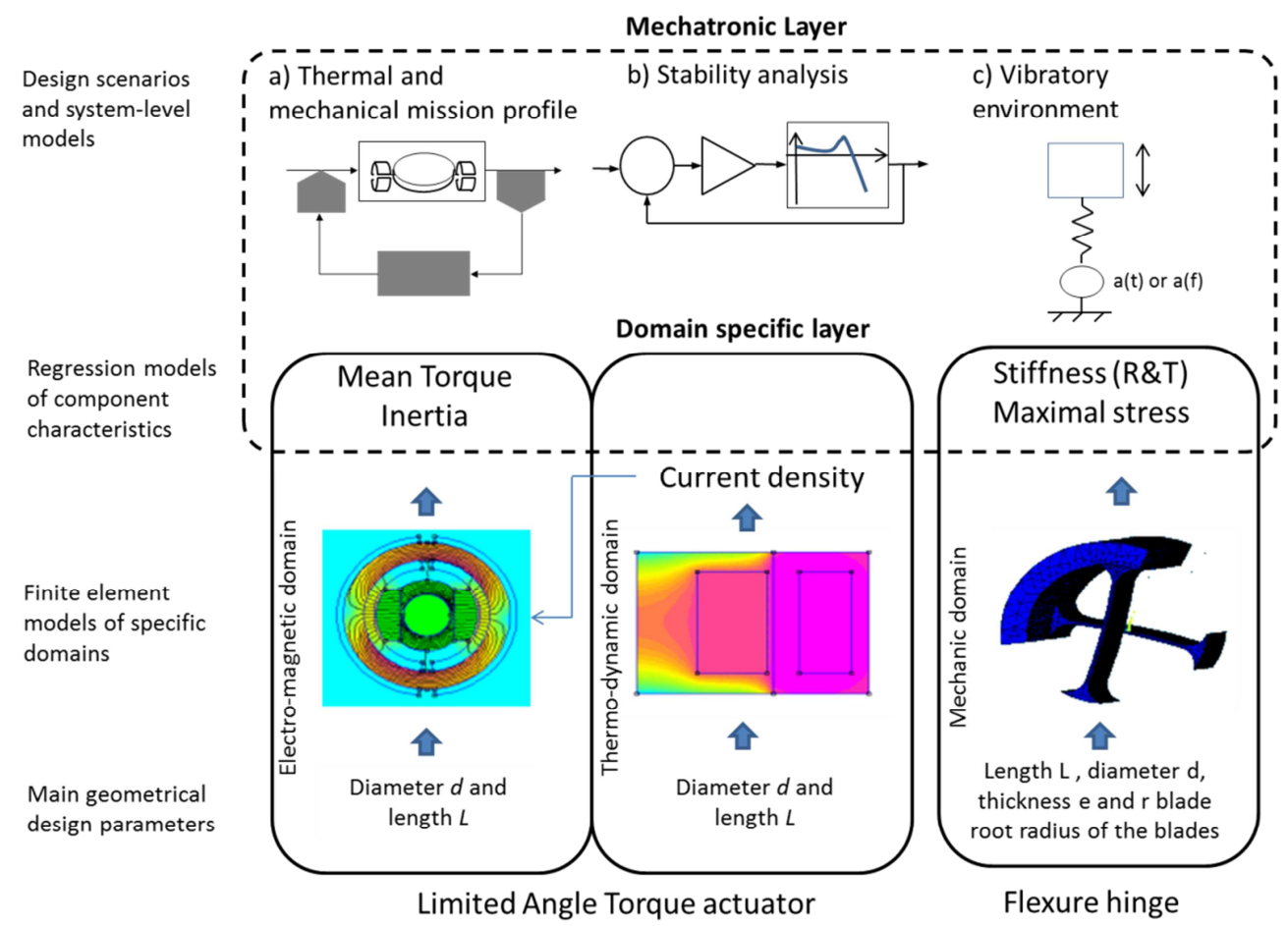

Figure 12 - Estimation models : interface between mechatronic and domain specific layers

To manage all these constraints and iterate quickly between mechatronic and domain specific layers during preliminary sizing, it may be interesting to have estimation models expressing, for example:

- for the LAT actuator, torque or inertia depending on the dimensions.

- for the flexure hinge, stiffness (in translation or in rotation), maximal stress depending on the dimensions.

The following paragraphs show how these models can be obtained using the regression process presented in this article. The results of scaling-law-based metamodels are compared to polynomial approximations [24] or Radial Basis Functions (RBF) [41] and kriging [27]. 


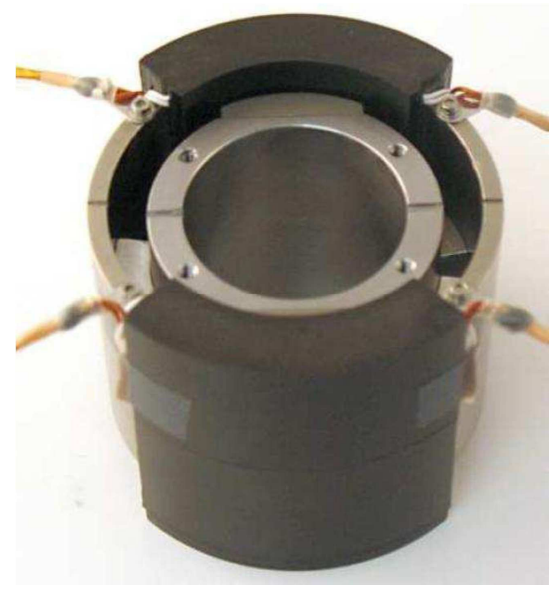

a) Limited Angle Torque (LAT) actuator (source : [37]): thermal and electromagnetic domains

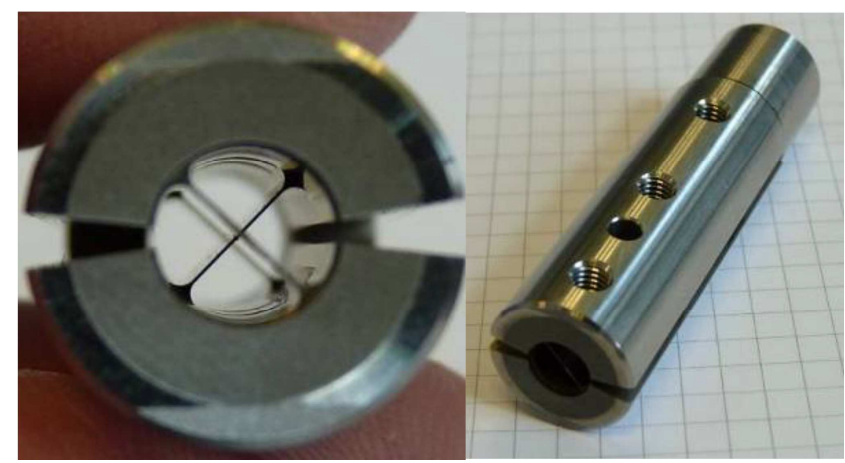

b) Flexural hinge (source [38]): mechanical domain

Figure 13 - Mechatronics component from different domains

\subsection{Limited angle actuator study}

The objective here was to illustrate how it is possible to link the continuous torque $T$ to the main dimensions, the length, $L$, and the diameter, $d$, of the actuator. All radial dimensions were geometrically similar to $d$. With such a choice, one has two design parameters influencing significantly the torque and inertia of the actuator, two main characteristics for the system level design, without representing all dimensional parameters used during the component level design. The desired equation thus took the following form:

$$
T=f\left(L, \pi_{1}\right)=k\left(\pi_{1}\right) L^{a\left(\pi_{1}\right)} \text { with } \pi_{1}=\frac{d}{L}
$$

The design code, to which the regressions were applied, was set up using two 2D finite element simulations (Figure 14) with FEMM software [42]. A first thermal simulation found the permissible current density $J$ for static continuous operation. The thermal environment was modeled by convective heat transfer between the periphery of the actuator and the environment. A second electromagnetic simulation used the previously calculated current density in order to obtain the continuous torque.

Several cases illustrate the regression method:

- The winding was moulded with or without a potting resin that ensured better conductive thermal transfer. This point addressed the effect of assumption c. (subsection 3.1). An equivalent thermal conductivity of copper and resin or air was deduced from a homogenization of the winding [43] [44].

- Two DoE for different ranges were used to test the robustness and validity of the regressions obtained outside their original design. The two DoE had 4 levels for $L$ and 6 levels for $\pi_{l}$ which led to 24 experiments. The difference between the DoE came from their range: for L, $[20 ; 60] \mathrm{mm}$ for 
the first DoE and [10;100] mm for the second, and for $\pi_{l},[0.5 ; 2.5]$ for the first DoE and [0.1;5] for the second.

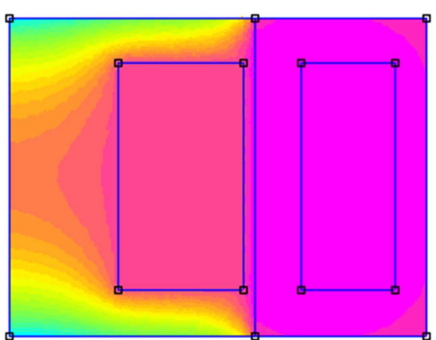

a) Thermal simulation

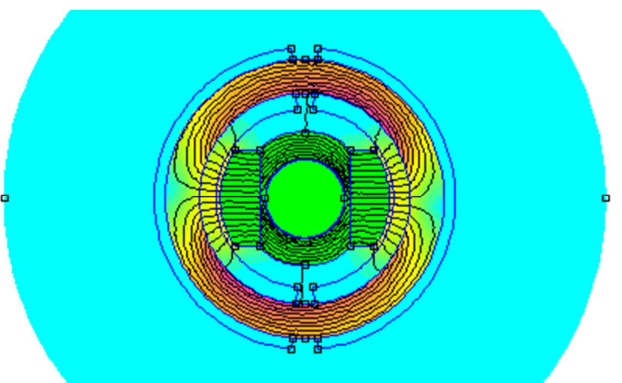

b) Electromagnetic simulation

Figure 14-2D finite element simulations of the LAT actuator

Figure 15 illustrates the step 2.1 of the process for the cases of winding with or without potting. In the case of winding with potting, the power coefficient varies between 3.42 and 3.49. Without potting, this coefficient varies between 3.23 and 3.48. The 2 cases have a power coefficient varying between 3.5 (demonstrated in section 3.2), characteristic of purely convective thermal transfer, and 3, characteristic of a purely conductive thermal transfer. A coil without potting is naturally more influenced by the conductive effect. For both cases, it was interesting not to consider parameter $a\left(\pi_{1}\right)$ as a constant.

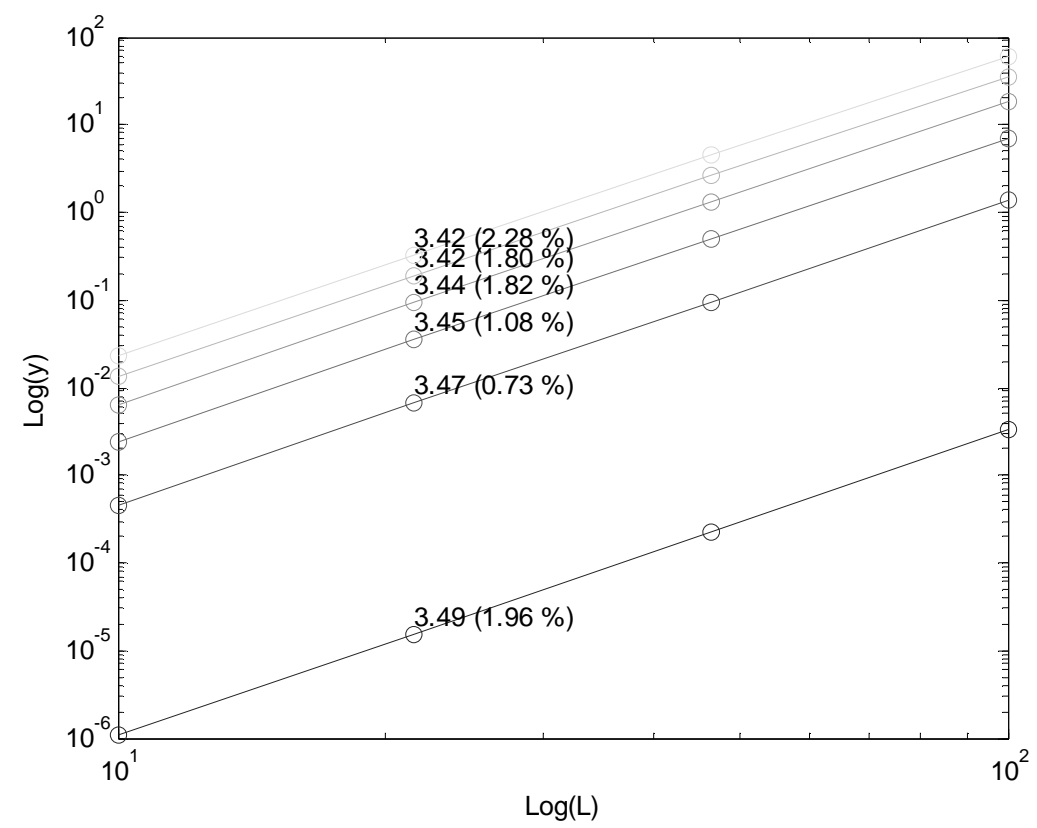

a) With potting resin 


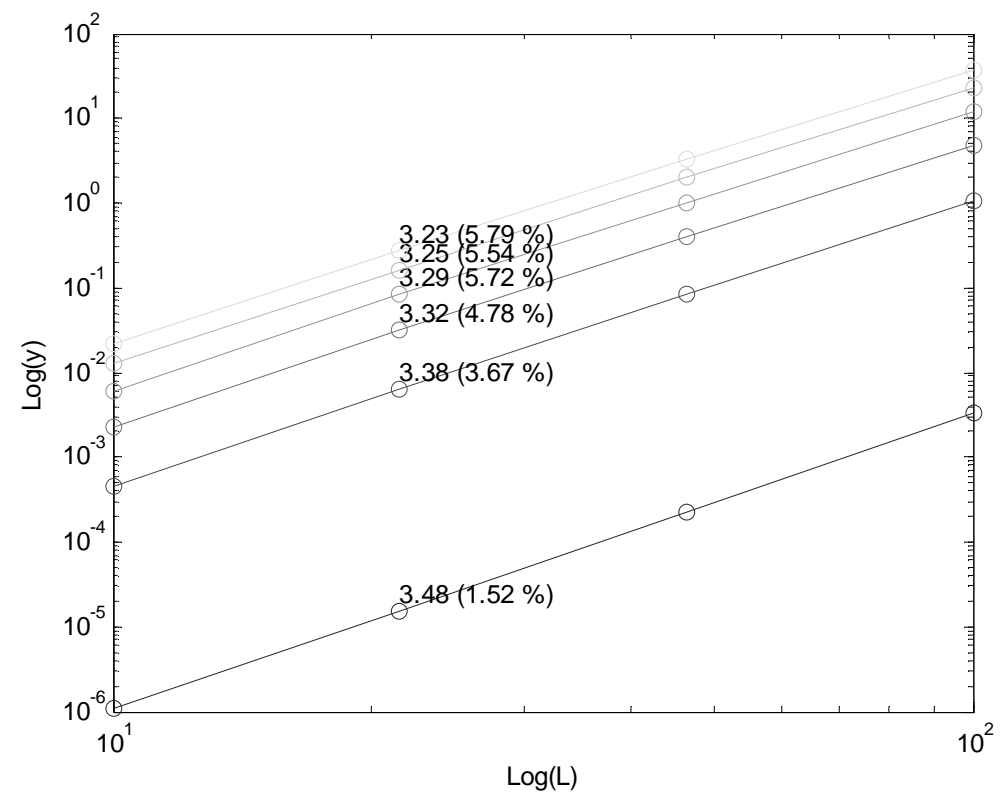

b) Without potting resin

Figure 15 - Effect of conductive thermal transfer on the power coefficient

Next, the proposed approach was applied to a winding with potting. The form of the scaling-law-based metamodel (SLAWMM) was:

$$
T=k\left(\pi_{1}\right) L^{a\left(\pi_{1}\right)}=c_{1} \pi_{1}^{c 2} L^{c_{3}+c_{4} \pi_{1}+c_{5} \pi_{1}^{2}}
$$

It was compared to three other approximations depending on the two design variables $L$ and $d$ : a $3^{\text {rd }}$ order polynomial response surface (RSM) [24], a Gaussian Radial Basis Function (RBF) [41] and a kriging approximation [26]. The polynomial functions were built with the same DoE as the scaling-law-based metamodels. The RBF and kriging approximations were built with a specific DoE: a latin hypercube (LHC) with the same range and number of experiments as the scaling-law-based metamodels, in this case 24 . The polynomial regression, RBF and kriging functions were obtained with $L$ and $d$ parameters and not with $L$ and $\pi_{l}$ which would not have been such a good choice. The least square regression for polynomial functions has minimized the relative error and not the absolute error in order to avoid gross errors on small actuators. The Gaussian width factor of RBF was chosen by minimizing a cross validation error [41]. The kriging approximation used a quadratic regression model and a Gaussian correlation function.

Figure 16 illustrates step 3.3, the model quality check, of the regression process. These validations were performed by comparing the 48 simulated results, 24 for the SLAWMM DoE and 24 for the LHC DoE, with the estimated results using each metamodel. Each approximation was thus tested with 24 configurations of the initial DoE that served for their construction and 24 other configurations within the same range. Note that the DoE using range 2 was much wider than when using range 1 , leading to four additional orders of magnitude of variations on the output quantity that was being approximated. In all cases (range 1 and range 2), the scalinglaw-based metamodels (SLAWMM) showed good prediction qualities: less than 2 and 10\% of maximum error for the range 1 (Figure 16a) and range 2 (Figure 16b) regression. The expressions obtained for equation (18) differ slightly between the 2 ranges (see figure captions). Polynomial approximations, on the other hand, even with twice number of coefficients to be determined, showed a larger maximum error : 3\% (Figure 16a) and 79\% (Figure 16b) for range 1 and 2 respectively. For range 2, the maximum error was $18.5 \%$ at the points of the DoE used for polynomial response surface construction but increases to $79 \%$ with the 24 configurations of the DoE that did not serve for its construction (predictive capability). Radial basis functions and kriging led to even worse results. These two metamodels had difficulty in coping with the very wide range of variation of the output quantity, which led, in particular to large errors for very small values. 
When the predictive capability of metamodels was assessed by testing them at points well outside their construction bounds, regression-based scaling laws remained valid (less than $15 \%$ error in Figure 16c). Gaussian RBF and kriging functions, with their high flexibility enabling a multiple peaks surface response to be represented, were at a disadvantage in this case of fairly monotonous surfaces: they could give very poor results at points involving large extrapolation. All three classic regression techniques (RSM, RBF and kriging) gave very poor results, even negative torque for small actuators, when they were used well outside the points that served for their construction (equations of range 1 used into range 2 as illustrated in Figure 16c).

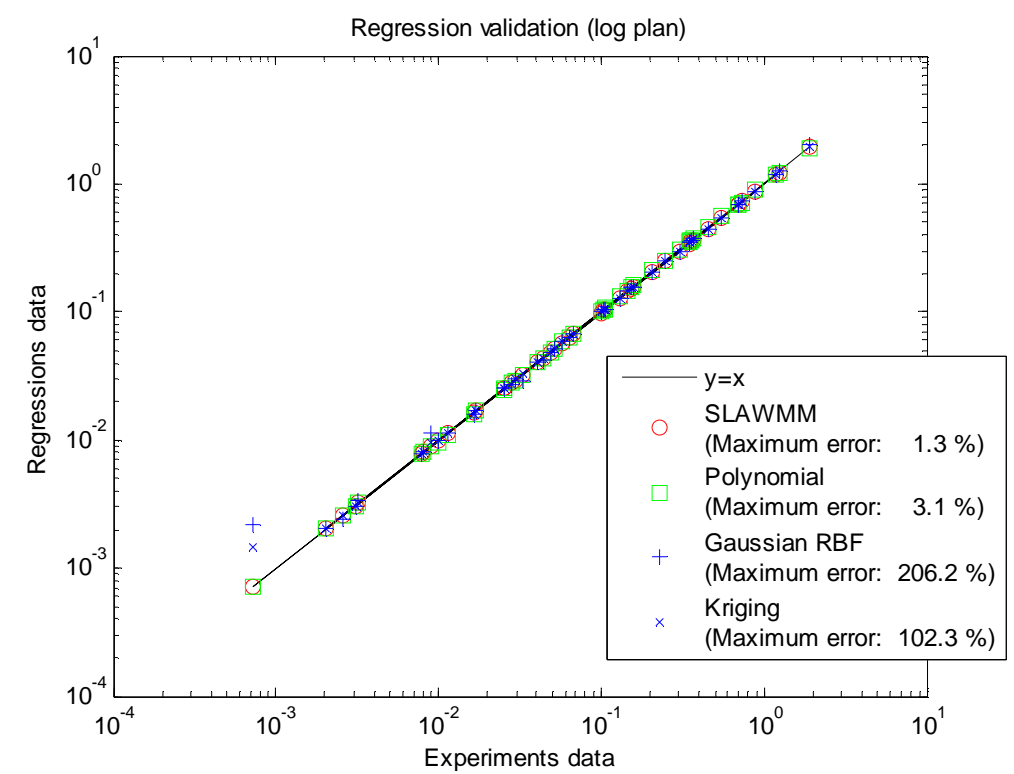

$T=k\left(\pi_{1}\right) L^{a\left(\pi_{1}\right)}=1.29 .10^{-7} \pi_{1}^{2.596} L^{3.487-0.021 \pi_{1}+0.003 \pi_{1}^{2}} \quad(T$ in N.m, $L$ in mm $)$

a) Range $1: L \in[20 ; 60] \mathrm{mm}$ and $\pi_{l} \in[0.5 ; 2.5]$

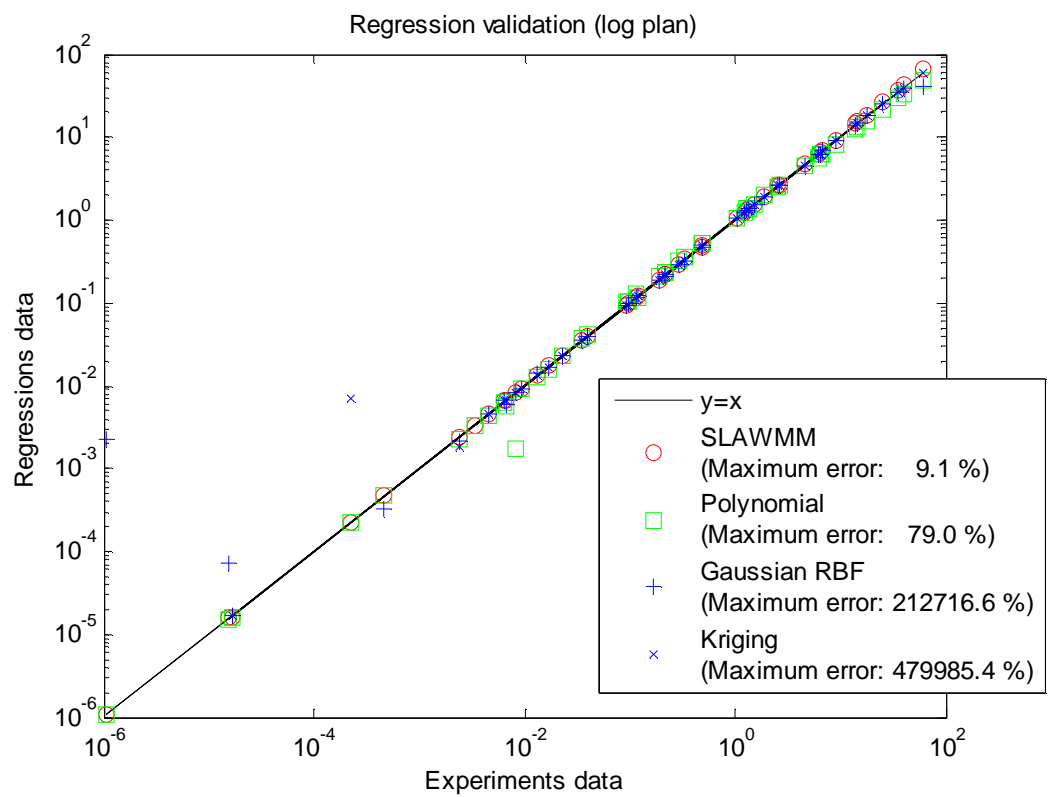

$T=k\left(\pi_{1}\right) L^{a\left(\pi_{1}\right)}=1.35 .10^{-7} \pi_{1}^{2.569} L^{3.476-0.016 \pi_{1}+0.002 \pi_{1}^{2}} \quad(T$ in N.m, $L$ in mm $)$

b) Range $2: L \in[10 ; 100] \mathrm{mm}$ and $\pi_{1} \in[0.1 ; 5]$ 




c) Equations from Range 1 used for Range 2

Figure 16 - Quality check of the regressions for different range of study

Power law forms are thus interesting as "structural components" for the specific metamodels of sizing problems where geometrical dimensions can have a wide range of variation. This robustness property can be important when conducting explorative design studies in areas not originally planned. As the regression form has a physical basis, some coefficients can be be fixed after a first complete study. For example, the $c_{3}$ coefficient of equation (27) can be fixed at 3.5 (see section 3.2) and future FEM simulations with different configurations have to find just 4 coefficients $\left(c_{1}, c_{2}, c_{4}\right.$ and $\left.c_{5}\right)$ with a reduced size DOE.

\subsection{Flexural hinge study}

The objective here was to illustrate how it is possible to link the rotational stiffness, $K_{r}$, to the main dimensions: $L$ the length, $d$ the diameter, $e$ the thickness, and $r$ the blade root radius (Figure 17a) of a flexural hinge composed of 2 thin blades of titanium alloy. The desired equation thus took the following form:

$$
\begin{gathered}
K_{r}=f\left(d, \pi_{1}, \pi_{2}, \pi_{3}\right)=k\left(\pi_{1}, \pi_{2}, \pi_{3}\right) d^{a\left(\pi_{1}, \pi_{2}, \pi_{3}\right)} \\
\text { with } \pi_{1}=\frac{r}{d}, \pi_{2}=\frac{e}{d} \text { and } \pi_{3}=\frac{L}{d}
\end{gathered}
$$

The design code, to which the regressions were applied, is set up using a 3D finite element simulation (Figure 17b) with ANSYS software [45]. This static analysis gave the angular deflection for a given torque on the hinge, which, by ratio, gave the rotational stiffness. The meta-modelling process was applied to this data $K_{r}$ but could also have been applied to other quantities of interest for the design, such as the stress-to-deflection ratio or the radial stiffness. This regression problem involves more parameters than the previous application example and allows us to illustrate the interest of the multiplication coefficient form analysis (step 2.1 of the process given in Figure 5). 


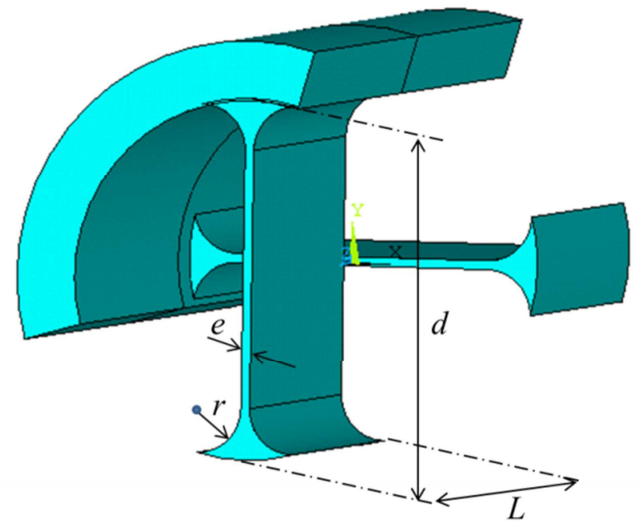

a) Hinge geometry

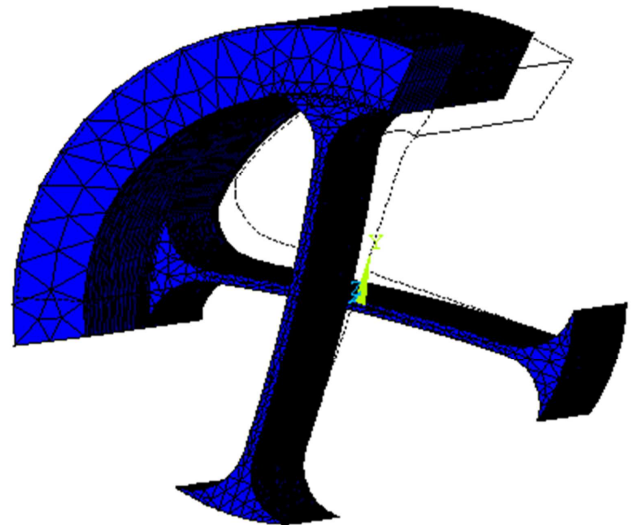

b) Flexural deformations of the hinge

Figure 17 - Geometry of the flexible hinge (2 blades)

The DoE used had 4 levels for $L$ and 3 levels for each $\pi_{i}$ parameters. The range studied was: $d \in[5 ; 50] \mathrm{mm}$, $\pi_{1} \in[0.05 ; 0.015], \pi_{2} \in[0.01 ; 0.04]$ and $\pi_{3} \in[0.5 ; 2]$. As illustrated by the result of step 2.1 (Figure 18), the power coefficient $a$ was constant and equal to 3 for any $\pi_{\mathrm{i}}$ parameters configurations. Thus, only one physical phenomenon drove the evolution of the rotational stiffness: the elasticity of the material. Figure 19, the result of step 2.1, shows that the influence of $\pi_{2}$ and $\pi_{3}$ parameters can be modeled by power laws expressions. The form of the $\pi_{1}$ function cannot be a power law and was modeled here by a polynomial response surface here. The form of the scaling-law-based metamodel was be thus:

$$
K_{r}=\left(c_{0}+c_{1} \pi_{1}+c_{2} \pi_{1}^{2}\right) \pi_{2}^{c 3} \pi_{3}^{c 4} d^{3}
$$

The regressions results were (with $K_{r}$ in N.m/rad and $d$ in $\mathrm{m}$ ) :

$$
K_{r}=\left(1.93 .10^{10}+3.10 .10^{10} \pi_{1}+2.30 .10^{11} \pi_{1}^{2}\right) \pi_{2}^{2.96} \pi_{3}^{1.05} d^{3}
$$

for the first one, where regression was carried out with $a$ and $k$ data (see step 3 section 3.2.3).

$$
K_{r}=\left(1.93 .10^{10}+3.10 .10^{2} \pi_{1}+2.30 .10^{11} \pi_{1}^{2}\right) \pi_{2}^{3} \pi_{3}^{1} d^{3}
$$

for the second one, carried out on the $y$ data with a least squares fit (see step 3 section 3.2.3).

For the last regression, powers of $\pi_{2}$ and $\pi_{3}$ parameters were set to 3 and 1 respectively. Equation (31) presents a simple form that can be handled and implemented in different calculation tools. As shown in Figure 20 , the relative error is controlled for a wide range of results with a maximum error of less than $15 \%$. These results are compared to a $3^{\text {rd }}$ order polynomial function here, which was the metamodel that performed the least poorly of the three alternatives (polynomial response surface, radial basis function, kriging) in the previous application example. The expression of the polynomial response surface required the determination of a large number of parameters: 26 parameters compared to 5 for the proposed form, and showed a maximum error of $21 \%$. The scaling-law-based metamodel allows light and compact models to be obtained, which can be easily integrated into other design algorithms. 


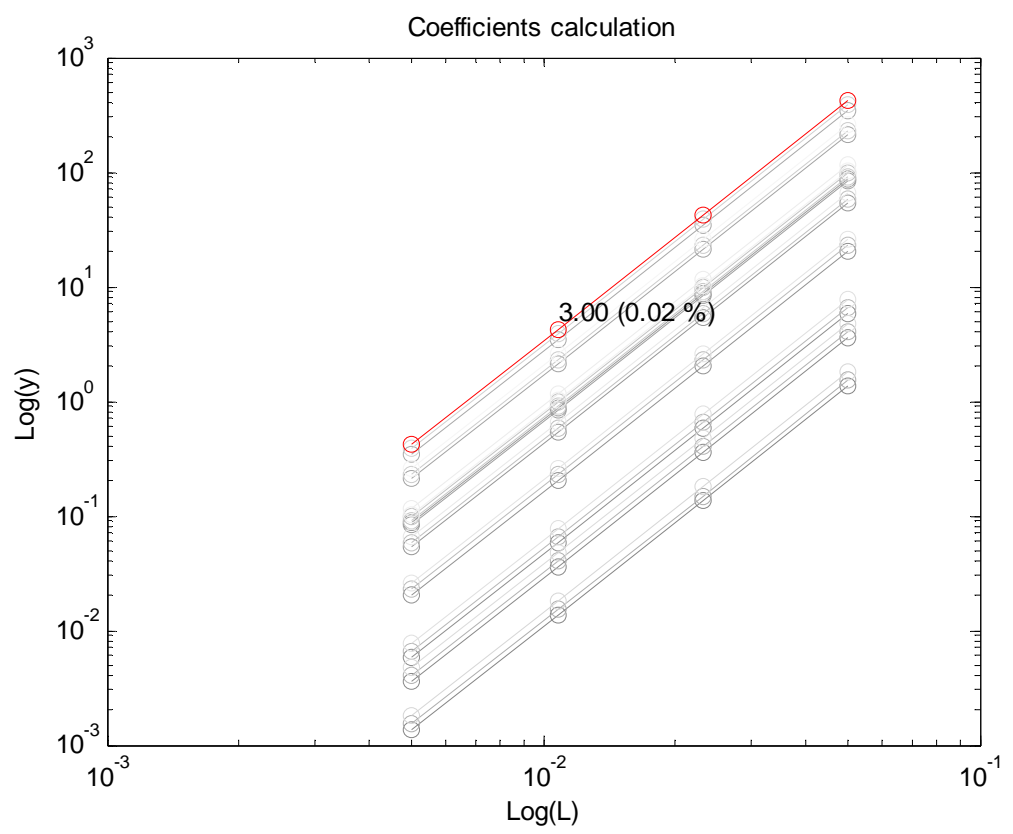

Figure 18 - Scaling laws with geometrical similarity

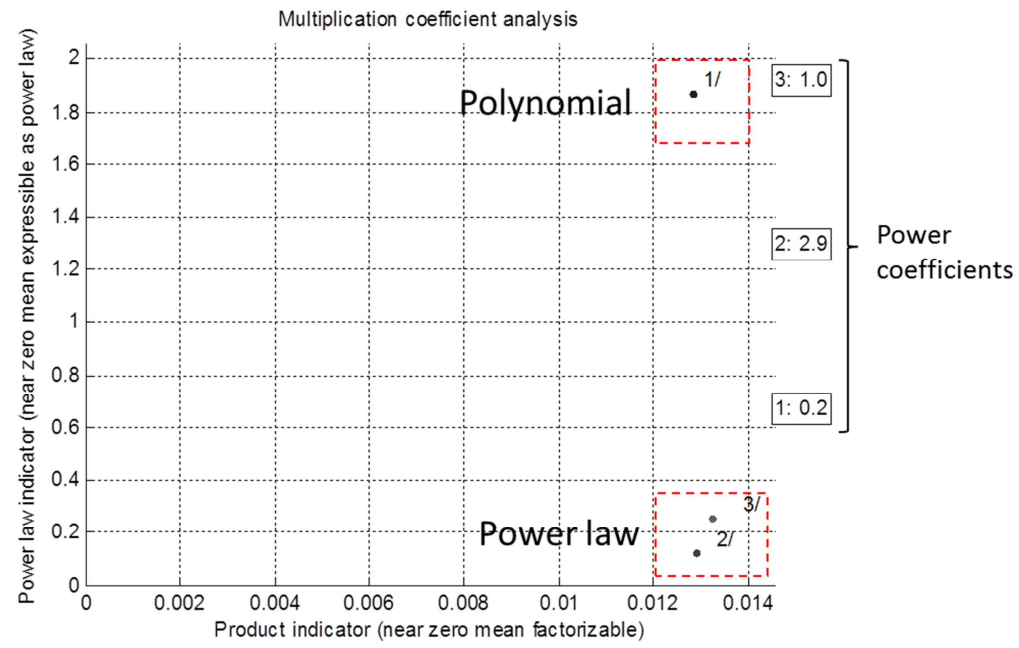

Figure 19 - Multiplication coefficient form analysis 


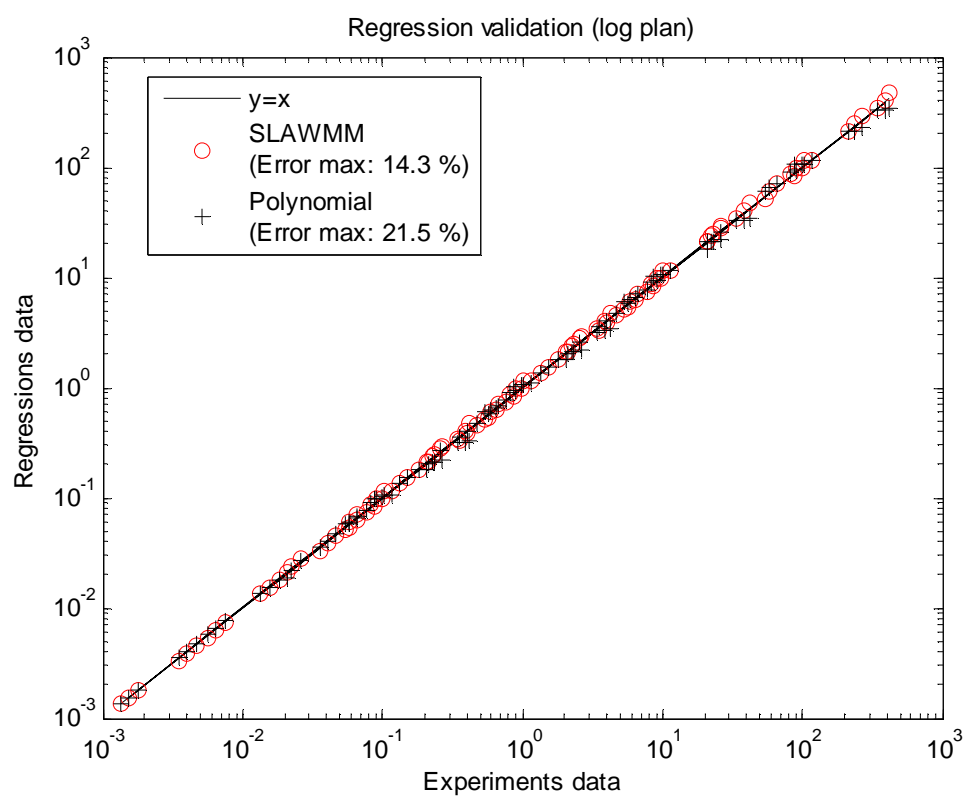

Figure 20 - Quality check of the regressions

\section{ConClusion}

The design of mechatronic systems requires estimation models to make the link between the design details of each component and the overall design of the system. The article has shown that scaling laws possess interesting properties for performing this role during the sizing tasks. A demonstration of the criteria for obtaining these scaling laws with the Buckingham theorem highlighted the assumptions required for this work. Two of these assumptions, geometric similarity and the uniqueness of the design criteria, limit the generality of their use. A method was proposed to overcome these limitations and generalize the form of scaling laws. The process of regression adapted to this form was described and validated on various examples. The regressions obtained were compared to classical metamodels and showed the interest of the physical basis of scaling-law-based metamodels for preliminary sizing of mechatronic components: their shape is easy to handle while remaining valid over a wide range of sizes, even for prediction or extrapolation purposes. The proposed form of regression can be applied to various fields encountered in mechatronic systems and is illustrated here by simulations on thermal, electromagnetic and mechanical examples.

\section{REFERENCES}

[1] VDI-Richtlinien, Design methodology for mechatronic systems. VDI, Düsseldorf..

[2] D. M. Auslander, "What is mechatronics?," Mechatronics, IEEE/ASME Transactions on, vol. 1, no. 1, pp. 5-9, Mar. 1996.

[3] H. Van der Auweraer, J. Anthonis, S. De Bruyne, and J. Leurdian, "Virtual engineering at work: the challenges for designing mechatronic products," Engineering with Computers, vol. 29, pp. 389-408, 2013.

[4] P. Hehenberger, F. Poltschak, K. Zeman, and W. Amrhein, "Hierarchical design models in the mechatronic product development process of synchronous machines," Mechatronics, vol. 20, pp. 864-875, 2010.

[5] F. Cellier and J. Greifeneder, Continuous System Modeling, Springer, Ed. 1991.

[6] F. Roos, H. Johansson, and J. Wikander, "Optimal selection of motor and gearhead in mechatronic applications," Mechatronics, vol. 16, no. 1, pp. 63-72, 2006.

[7] D. P. Raymer, Aircraft Design: A Conceptual Approach, Fourth Edition. AIAA Education Series, 2006.

[8] M. Budinger, J. Liscouët, F. Hospital, and J.-C. Maré, "Estimation models for the preliminary design of 
electromechanical actuators," Proceedings of the Institution of Mechanical Engineers, Part G: Journal of Aerospace Engineering, vol. 226, no. 3, pp. 243-259, 2012.

[9] J. Liscouet, M. Budinger, J.-C. Maré, and S. Orieux, "Modelling approach for the simulation-based preliminary design of power transmissions," Mechanism and Machine Theory, vol. 46, no. 3, pp. 276-289, 2011.

[10] M. Budinger, R. Reysset, T. El Halabi, C. Vasiliu, and J.-C. Mare, "Optimal preliminary design of electromechanical actuators," Proc IMechE Part G: J Aerospace Engineering, To be published.

[11] O. Gomis-Bellmunt, F. Campanile, S. Galceran-Arellano, D. Montesinos-Miracle, and J. Rull-Duran, "Hydraulic actuator modeling for optimization of mechatronic and adaptronic systems," Mechatronics, vol. 18, no. 10, pp. 634640, 2008.

[12] O. Gomis-Bellmunt, S. Galceran-Arellano, A. Sudrĩ̃ -Andreu, D. Montesinos-Miracle, and L. F. Campanile, "Linear electromagnetic actuator modeling for optimization of mechatronic and adaptronic systems," Mechatronics, vol. 17, no. 23, pp. 153-163, 2007.

[13] G. Pahl and W. Beitz, Engineering Design: A Systematic Approach, Springer, Ed. 2007.

[14] R. Bialecki, A. Kassab, and A. Fic, "Proper orthogonal decomposition and modal analysis for acceleration of transient FEM thermal analysis," Int J Numer Methods Eng, vol. 62, p. 774-797, 2005.

[15] D. Ryckelynck, F. Chinesta, E. Cueto, and A. Ammar, "On the a priori model reduction: overview and recent developments," Archives on Computational Methods in Engineering, vol. vol 13(1), p. 91-128, 2006.

[16] T. Simpson, J. Peplinski, P. Koch, and J. Allen, "“Metamodels for Computer-Based Engineering Design: Survey and Recommendations," Engineering with Computers, vol. 17, pp. 129-150, 2001..

[17] S.-U. Chung, et al., "Design and experimental validation of doubly salient permanent magnet linear synchronous motor for precision position control," Mechatronics, no. 0, pp. -, 2013.

[18] F. Chinesta, et al., "PGD-Based Computational Vademecum for Efficient Design, Optimization and Control," Archives of Computational Methods in Engineering, vol. Volume 20, Issue 1, pp. 31-59, 2013.

[19] E. S. Taylor, Dimensional Analysis for Engineers. Oxford University Press, 1974.

[20] G. Murphy, Similitude in engineering. The Ronald press company. New York, 1950.

[21] G. Miragliotta, "The power of dimensional analysis in production systems design," Int. J. Production Economics, vol. 131, pp. 175-182, 2011.

[22] M. Pfenning, F. Thielecke, and U. B. Carl, "Recent Advances Towards an Integrated and Optimized Design of High-lift Actuation Systems," in SAE Int. J. Aerosp. 3(1):55-64, 2010, doi:10.4271/2009-01-3217., 2010.

[23] M. D. Petersheim and S. N. Brennan, "Scaling of hybrid-electric vehicle powertrain components for Hardware-inthe-loop simulation," Mechatronics, vol. 19, p. 1078-1090, 2009.

[24] H. M. Raymond, D. C. Montgomery, and M. A.-C. Christine, Response surface methodology : process and product optimization using designed experiments, 4th ed. John Wiley \& Sons, 2006.

[25] R. Jin, W. Chen, and T. W. Simpson, "Comparative studies of metamodelling techniques under multiple modelling criteria," Struct Multidisc Optim, vol. 23, pp. 1-13, 2001.

[26] M. Stein, Interpolation of Spatial Data: Some Theory for Kriging. Springer (New York), 1999.

[27] J. Kleijnen, "Kriging metamodeling in simulation: A review," European Journal of Operational Research, vol. Vol. 192, No. 3, pp. 707-716, 2009.

[28] R. Damon and W. Harvey, Experimental design, ANOVA, and regression. Harper \& Row (New York), 1987.

[29] I. Sobol, "Sensitivity estimates for non-linear mathematical models," Math Modeling Comput Exp, vol. 4, p. 407414, 1993.

[30] M. D. Morris, "Factorial Sampling Plans for Preliminary Computational Experiments," Technometrics, vol. 33, issue $\mathrm{n}^{\circ} 2$, pp. 161-174, 1991.

[31] C. Gogu, R. T. Haftka, S. K. Bapanapalli, and B. V. Sankar, "Dimensionality Reduction Approach for Response Surface Approximations: Application to Thermal Design," AIAA Journal, vol. 47, no. 7, pp. 1700-1708, 2009.

[32] G. A. Vignaux and J. L. Scott, "Simplifying regression models using dimensional analysis," Austral. $\backslash \&$ New Zealand J. Statist., vol. 41, pp. 31-41, 1999.

[33] D. Lacey and C. Steele, "The Use of Dimensional Analysis to Augment Design of Experiments for Optimization and Robustification," Journal of Engineering Design, vol. Vol. 17, No. 1, p. 55-73, 2006.

[34] P. Mendez and F. Ordonez, "Scaling laws from statistical data and dimensional analysis," Journal of Applied 
Mechanics, vol. Volume 72, Issue 5, pp. 648-658, 2005.

[35] D. F. Young, "Basic principles and concepts of model analysis," Experimental Mechanics, vol. Volume 11, , Issue 4, pp. 325-336, 1971

[36] C. Tsai, S. Lin, H. Huang, and Y. Cheng, "Design and control of a brushless DC limited-angle torque motor with its application to fuel control of small-scale gas turbine engines," Mechatronics, vol. 19, pp. 29-41, 2009.

[37] N. Bencheikh, R. Le Letty, F. Claeyssen, G. Magnac, and G. Migliorero, "Limited angel torque actuator for fine angular positioning," in Proceedings of the 12th International Conference on New Actuators, Actuator 2010, Bremen, Germany, 14-16 june, 2010, 2010.

[38] I. Santos, I. Ortiz de Zárate, and G. Migliorero, "High accuracy flexural hinge development," in Proceedings of the 11th European Space Mechanisms and Tribology Symposium, ESMATS 2005, 21-23 September 2005, Lucerne, Switzerland., 2005.

[39] Q. Zhou, P. Ben-Tzvi, D. Fan, and A. A. Goldenberg, "Design of Fast Steering Mirror systems for precision laser beams steering," in Robotic and Sensors Environments, 2008. ROSE 2008. International Workshop on, Oct. 2008, pp. 144-149.

[40] J. Vinals, et al., "MTG scan mechanism predevelopment: design \\& performance," in '14th European Space Mechanisms \\& Tribology Symposium - ESMATS 2011', Constance, Germany, 28-30 September 2011, 2011.

[41] A. Forrester, A. Sóbester, and A. J. Keane, Engineering design via surrogate modelling: a practical guide. J. Wiley, 2008.

[42] D. Meeker, "Finite Element Method Magnetics Software," www.femm.info.

[43] L. Idoughi, X. Mininger, F. Bouillault, L. Bernard, and E. Hoang, "Thermal Model With Winding Homogenization and FIT Discretization for Stator Slot," Magnetics, IEEE Transactions on, vol. 47, no. 12, pp. 4822-4826, Dec. 2011.

[44] W. T. Perrins, D. R. McKenzie, and R. C. McPhedran, "Transport properties of regular arrays of cylinders," Pro. R. Soc. Lond, vol. A 369, pp. 207-225, 1979.

[45] ANSYS. www.ansys.com. 